\title{
Socioeconomic Patterning of Cardiovascular Disease and its Risk Factors among Indians: A Systematic Review of Literature
}

\author{
Arti Singh ${ }^{1 *}$ and Shikha Dixit ${ }^{2}$
}

\section{Arti Singh ${ }^{1 *}$ and Shikha Dixit $^{2}$}

${ }^{1}$ Department of Humanities and Social Sciences, Indian Institute of Technology Kanpur, Kanpur 208016, INDIA. ${ }^{2}$ Department of Humanities and Social Sciences, Indian Institute of Technology Kanpur, Kanpur 208016, INDIA.

Correspondence

\section{Arti Singh,}

Department of Humanities and Social Sciences, Indian Institute of Technology Kanpur-208016, INDIA.

Phone no. +91-512-2597157 (office)

E-mail: aartis@iitk.ac.in;

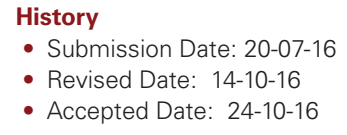

DOI : 10.5530/ijmedph.2017.1.1

Article Available online http://www.ijmedph.org/v7/i1

\section{Copyright}

(C) 2017 Phcog.Net. This is an openaccess article distributed under the terms of the Creative Commons Attribution 4.0 International license.

\begin{abstract}
Objective: To investigate the socioeconomic patterning of cardiovascular disease (CVD), its mortality and associated risk factors in the Indian population. Methods: Studies conducted on Indian population between January 1992 and the second week of April 2015 satisfying predefined inclusion and exclusion criteria were selected. PubMed, Ebscohost, Google scholar and Google were searched for CVD prevalence, CVD mortality and its six risk factors (alcohol, tobacco, hypertension, diabetes, obesity and sedentary lifestyle w.r.t. socioeconomic status (SES)). Result: 3, 550, 404 participants and 1, 71, 657 households were studied though 72 selected studies. Of these 13, 31, 23, 20, 21 and 16 articles investigated alcohol, tobacco, hypertension, diabetes, obesity and sedentary lifestyle, respectively, and 11 studies investigated the trend of CVD and its mortality w.r.t. to SES. Higher SES is found to be positively associated with hypertension, diabetes, obesity and sedentary lifestyle, whereas lower SES is found to be positively associated with alcohol and tobacco consumption only. No consensus has been found among studies over socioeconomic patterning of CVD, but the burden of its mortality has been found to be positively associated with lower SES. Conclusion: Even after 25 years of liberalisation of the Indian economy, the association between the CVD and SES is still positive. Rich people are getting more affected by CVD risk factors, but the burden of CVD mortality lies with poor people who cannot afford expensive drugs and interventional treatment. Poor healthcare facilities, high out-of-pocket expenditure and not-so-favourable policies are adversely affecting the CVD health of the weaker sections of India. Key words: Cardiovascular (CVD) diseases, CVD risk factors, Socioeconomic disparity/inequality, India.
\end{abstract}

\section{INTRODUCTION}

Cardiovascular disease (CVD) is responsible for causing the highest number of deaths worldwide in the age group of 15-59 years, which is the most productive age-group population for any country. ${ }^{1}$ In India, $26 \%$ deaths annually have been attributed to this disease. ${ }^{2}$ Notably, in India, where poverty rate is $37.2 \%$ and which accounts for $20 \%$ of the world population living below poverty line, the role of SES becomes more important than other factors in placing the country on the top spot of CVD-affected countries followed by China and Russia. ${ }^{2,3}$ CVD, being a non-communicable disease, has been reported to be associated with societies having a higher socioeconomic status (SES). However, with economic development and epidemiological transition, people from lower SES are now getting more affected by CVD than their higher socioeconomic counterparts. ${ }^{4}$ The Indian society has also undergone an epidemiological transition due to economic liberalisation and development; lifestyle changes have occurred and facilities which were earlier exclusive only to higher SES groups are now available to the people of lower SES also. ${ }^{5}$ Consequently, a reversal of social gradient has been reported by many researchers, i.e. morbidity and mortality due to CVD have increased in the lower SES group. ${ }^{4-6}$

In an early study on the urban population of Chandigarh, India, CVD has been reported to increase with
SES. ${ }^{7}$ Similarly, a positive association between the SES and CVD has been reported by other researchers too..$^{8-12}$ On the other hand, some studies have also reported a negative association and have concluded that the CVD mortality rate is higher in lower SES groups. ${ }^{6,13-16}$ However, Subramanian et al. have reported a higher prevalence of CVD risk factors in high SES groups, but a higher death rate among lower SES groups. ${ }^{17}$ They also suggested that many studies have wrongly concluded that the pattern of CVD in India is now similar to that in developed countries. Though their review has sparked a fresh debate over the role of SES in the development of CVD in India, they included studies prior to the economic liberalisation of 1991; this inclusion may have affected the conclusion regarding the relationship between the SES and CVD. ${ }^{18,19}$ Moreover, they excluded the urban-rural (U/R) difference as an SES marker. Many researchers have supported the inclusion of $U / R$ differences as an SES marker, because in India these differences play an important role in education, occupation, income, standard of living and social structure..$^{20,21}$

The aim of the present review is to improve our understanding of the pattern of CVD in India stratified by SES and to provide an update on how SES influences cardiovascular risk factors (CVRFs) and CVD mortality in the Indian population after 25 years of economic liberalisation. This review has used various SES markers in addition to $U / R$ differences and has 
focused only on selected modifiable and 'major established risk factors' of CVD identified by WHO (2004)2 namely, tobacco, alcohol, hypertension, obesity, sedentary lifestyle and diabetes.

\section{METHODS}

\section{Search Strategy}

Studies were searched from PubMed, Google Scholar, Ebscohost and Google. Citation and reference lists of all selected articles were further examined to obtain relevant articles. The aim is to review the maximum number of studies on SES patterning irrespective of study design, as only a limited number of studies are available on the topic and exclusion of a study due to its study design would limit the conclusions of the present review.

Studies conducted after 1991 were selected as the liberalisation of the Indian economy started from 1991, resulting in major lifestyle changes. Relevant studies conducted on urban and/or rural population of India to investigate CVD and/or any of its risk factor of interest w.r.t. SES were included. Information on the sample size, age group/mean age of the participants and criteria used for diagnosis were also used. All types of socioeconomic markers, including U/R differences, were selected.

Search included the combination of following terms: coronary heart disease, socioeconomic status, SES, India, cardiovascular diseases, coronary artery disease, risk factors, diabetes, hypertension, sedentary lifestyle, physical activity, alcohol, smoking, tobacco use, obesity, overweight, socioeconomic disparity/inequality, income level, educational level, blood pressure and sociodemographic factors.

\section{Inclusion and Exclusion Criteria for Study Selection}

Any empirical study that has investigated any of the above mentioned six risk factors w.r.t. any SES marker were included if their full text is accessible and if they were conducted between January 1992 to the second week of April 2015.

Studies carried out only on children and/or adolescents or on Indian migrants and reviews, letters, and articles not based on English language were excluded.

\section{Data Extraction}

After analysing the studies on the basis of the abovementioned inclusion and exclusion criteria, data were extracted from the selected studies in the form of authors' names, year of publication, study site, total sample, age group or mean age of the participants, marker of SES, diagnostic criteria for outcome variable/s, study design, outcome variables and the direction of their association with SES. All the reporting characteristics of the selected studies and the direction of association between CVD, CVRFs and various SES markers are listed in Table 1. The extracted data were reviewed by the second author separately and independently and disagreements were resolved after discussion. In order to avoid compli-
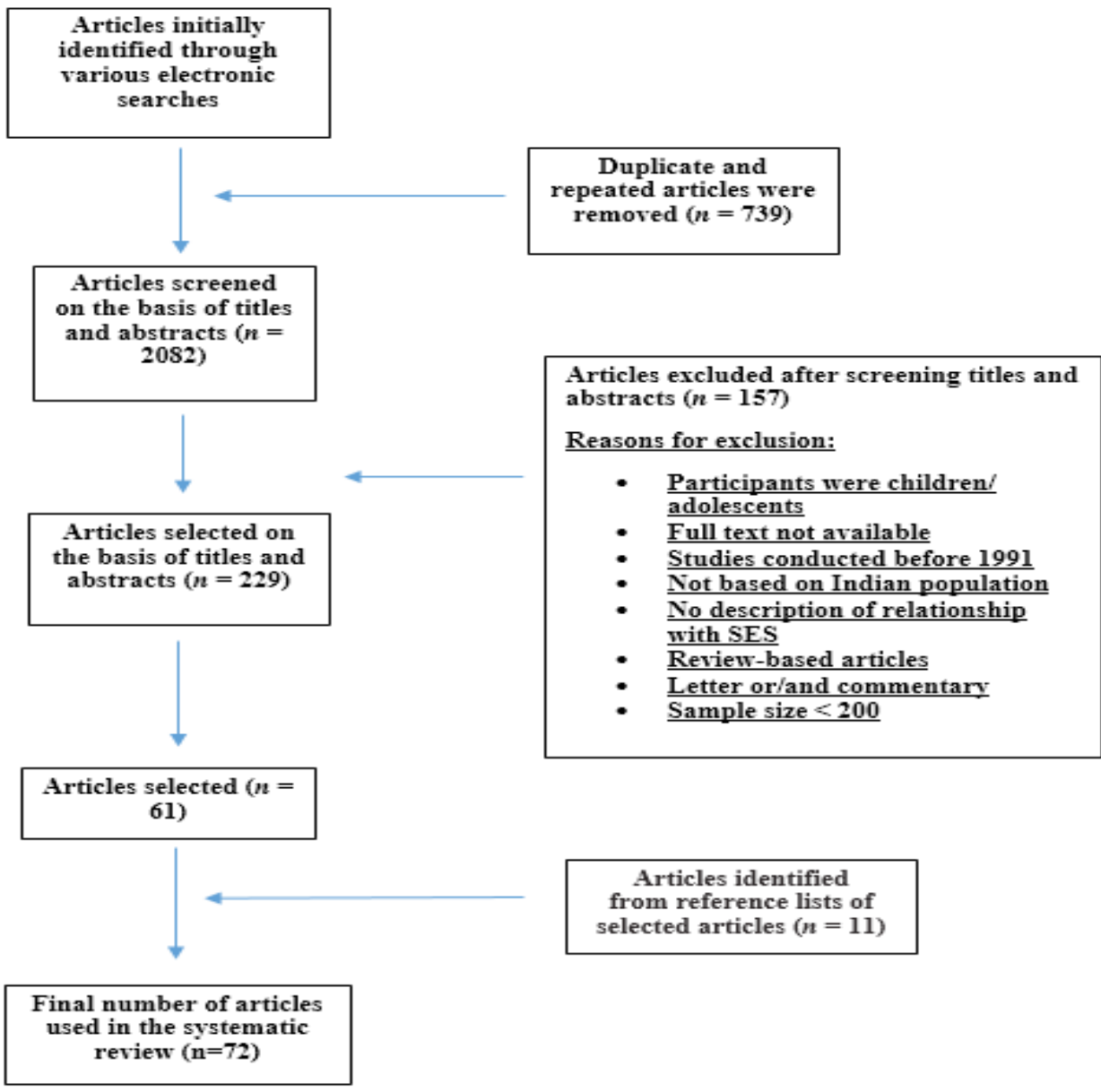

Duplicate and

repeated articles were

removed $(n=739)$

Figure 1: Flow diagram of the process of selection of studies. 
cations, the data were further analysed w.r.t. four most used SES markers only, namely education, income, residence and occupation.

\section{RESULTS}

\section{Search Result}

Our systematic review identified 229 records based on titles and abstracts, out of which 61 were selected for the final review as they fulfilled the inclusion criteria. Eleven additional studies were included after scanning the reference lists and citation lists of all articles.

\section{Study Characteristics}

Out of these 72 studies, 15 investigated tobacco, 5 diabetes, 7 obesity, 3 sedentary lifestyle, 9 alcohol, 5 hypertension, 5 CVD prevalence and 1 CVD mortality only, while the remaining 22 studies investigated a combination of these six risk factors and/or CVD prevalence and mortality w.r.t. SES. $83 \%$ studies were cross-sectional in our review. Total 3,550,404 participants and 1, 71, 657 households were investigated by the selected studies. Out of 72 studies, only 18 were conducted at the national level, while the remaining studies were conducted on one or more than one states or union territories of India. Also, 41 studies were conducted on single states, whereas 13 studies involved multiple states.
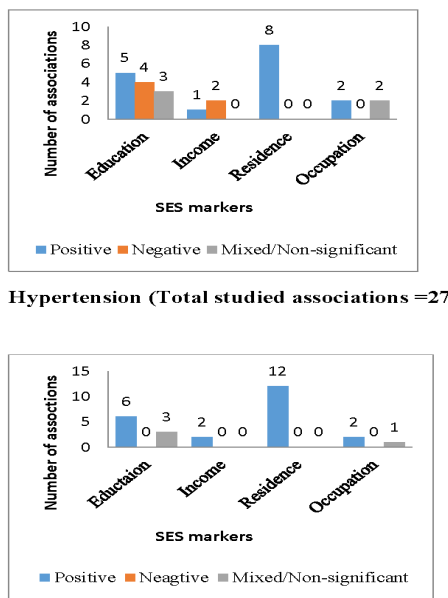

Obesity (Total studied associations $=26$ )

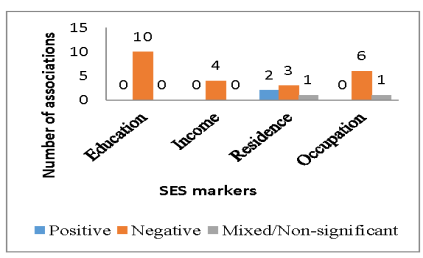

Alcohol (Total studied associations $=27$ )

\section{CVD Risk Factors across Socioeconomic Status}

\section{Hypertension}

The 23 studies $^{8,9,12-14,22-39}$ selected in this review to evaluate hypertension have used the same diagnostic criteria, which include the WHO crite$\mathrm{ria}^{25}$ as well as self-reported hypertensive cases with or without treatment. ${ }^{22,27,29}$ The trend of hypertension among Indians is different from that of developed countries, where the problem of hypertension clearly lies with lower socioeconomic population. 4 In India, there is no clear pattern for hypertension. Out of 27 associations (Figure 2) for the four most common SES markers (education, income, residence and occupation), 16 reported positive, 6 negative and 5 reported non-significant and mixed results with hypertension, suggesting a transitional phase through which India is going.

\section{Diabetes}

In total, 20 studies ${ }^{8,9,13,22,27,29-32,34,37-46}$ were selected for reviewing the relationship between the SES and diabetes. Diagnostic criteria were either based on the guidelines of ICMR, ${ }^{37}$ WHO, ${ }^{9}$ American Health Association $^{30}$ or on self-reported or known cases of diabetes. Out of 25 associations that we have examined w.r.t. education, income, residence and occupation, only one association reported negative, six reported nonsignificant or mixed results and the remaining reported a positive association (Figure 2). Contrary to Western countries, these results suggest that in India, diabetes is more prevalent among the affluent classes.

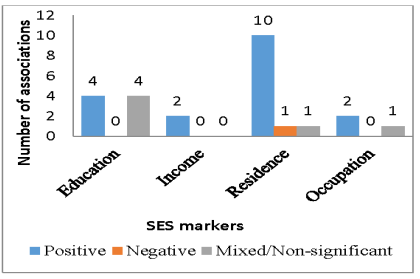

Diabetes (Total studied associations $=25$ )

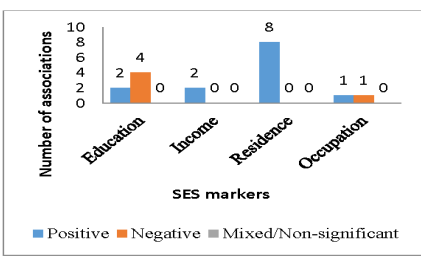

Sedentary Lifestyle (Total studied associations $=18$ )

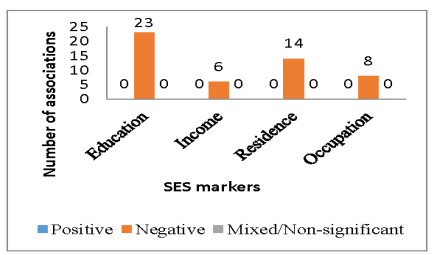

Tobacco $($ Total studied associations $=51)$

Figure 2 Graphs showing the number of positive, negative and mixed/non-significant associations reported in selected studies. Data analyzed for alcohol, tobacco, diabetes, hypertension, obesity and sedentary lifestyle w.r.t Reproduction size: Full page width

Figure 2: Graphs showing the number of positive, negative and mixed/non-significant associations reported in selected studies. Data analyzed for alcohol, tobacco, diabetes, hypertension, obesity and sedentary lifestyle w.r.t the four most used socioeconomic markers viz. education, income, residence and occupation. 


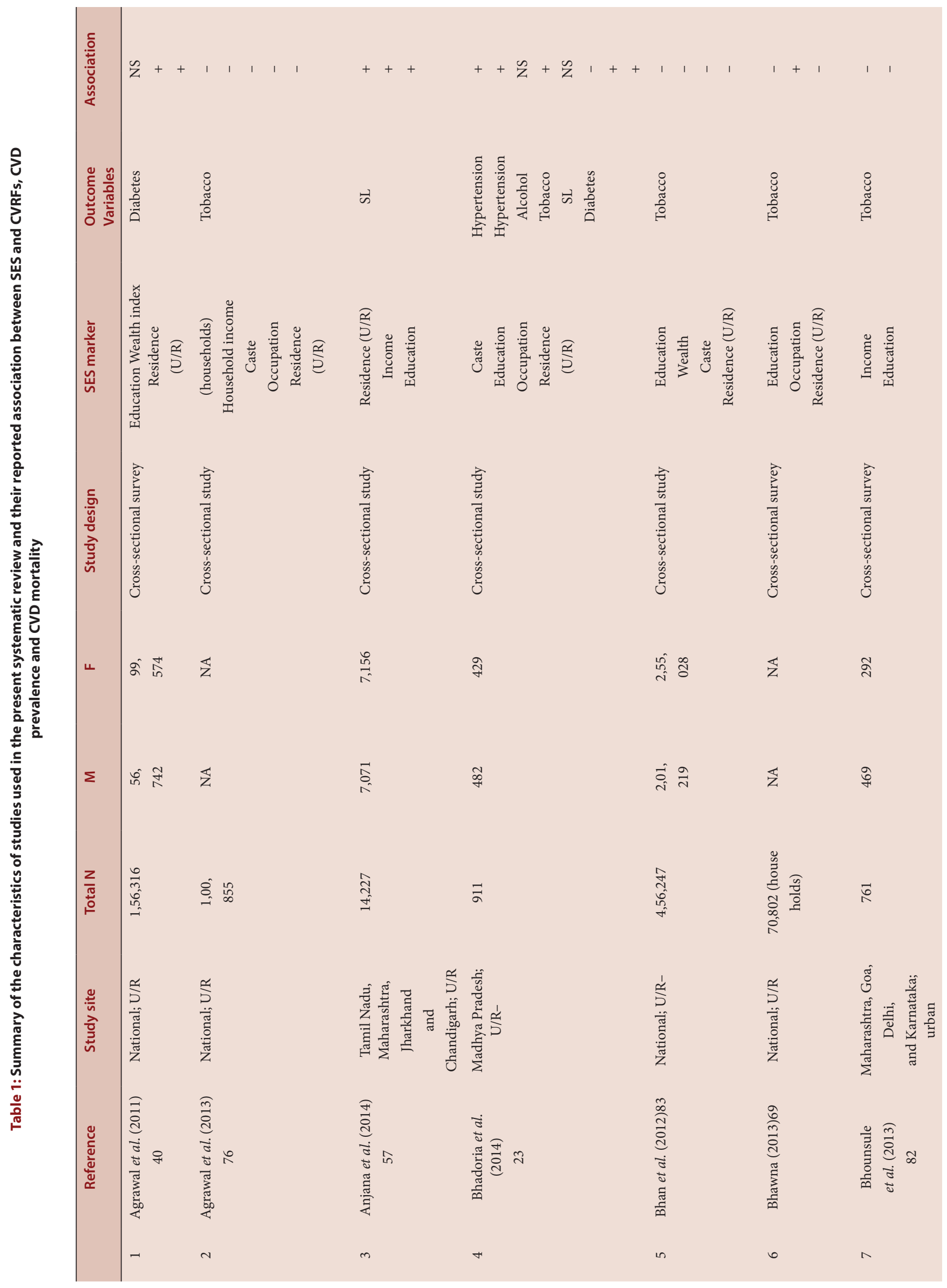




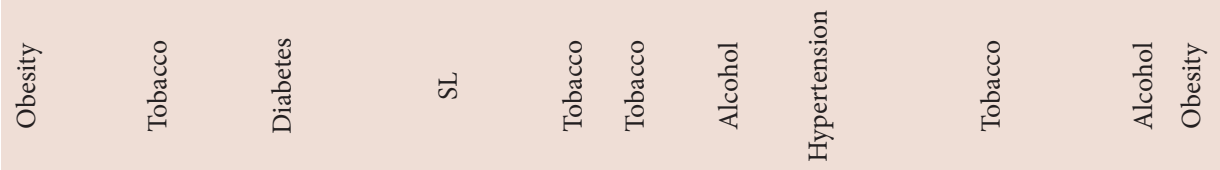

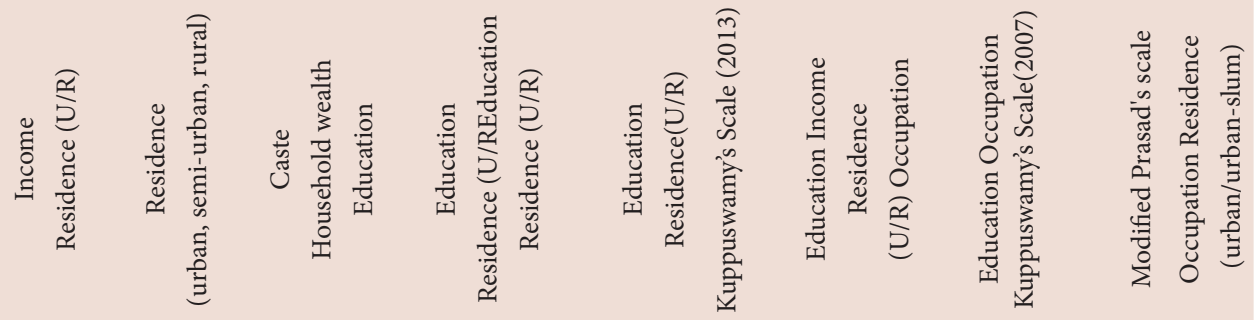

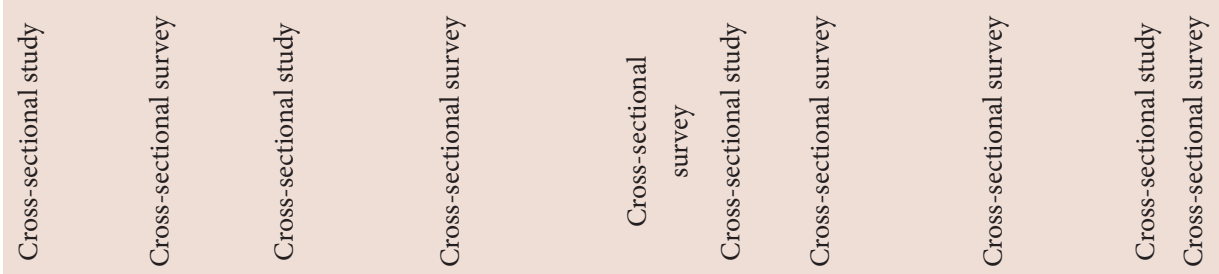

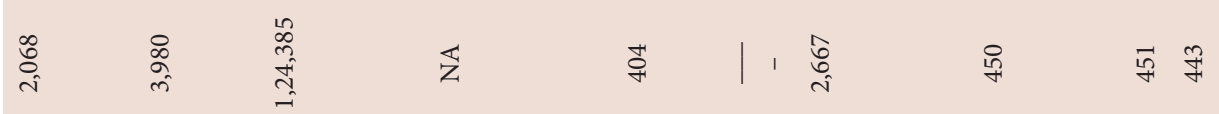

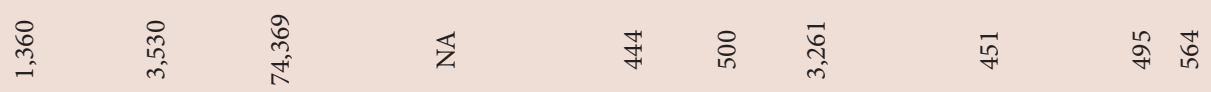

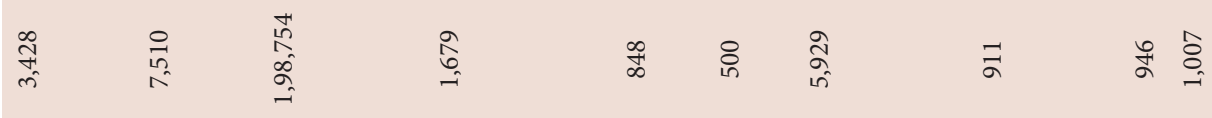

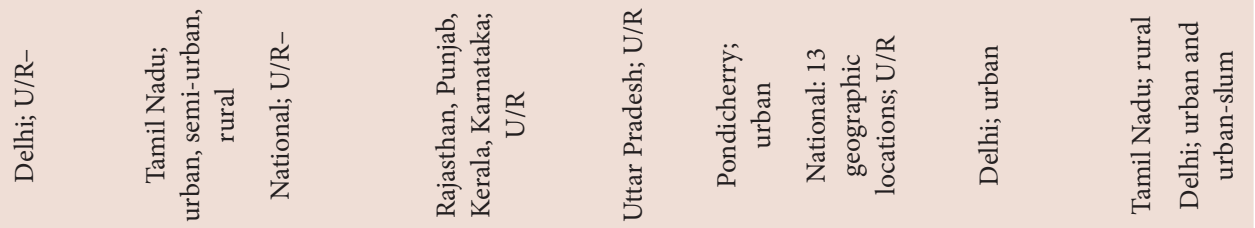

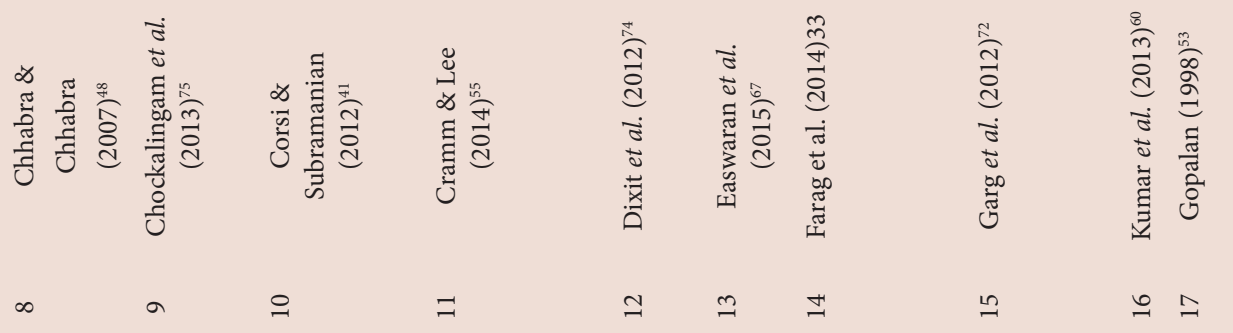




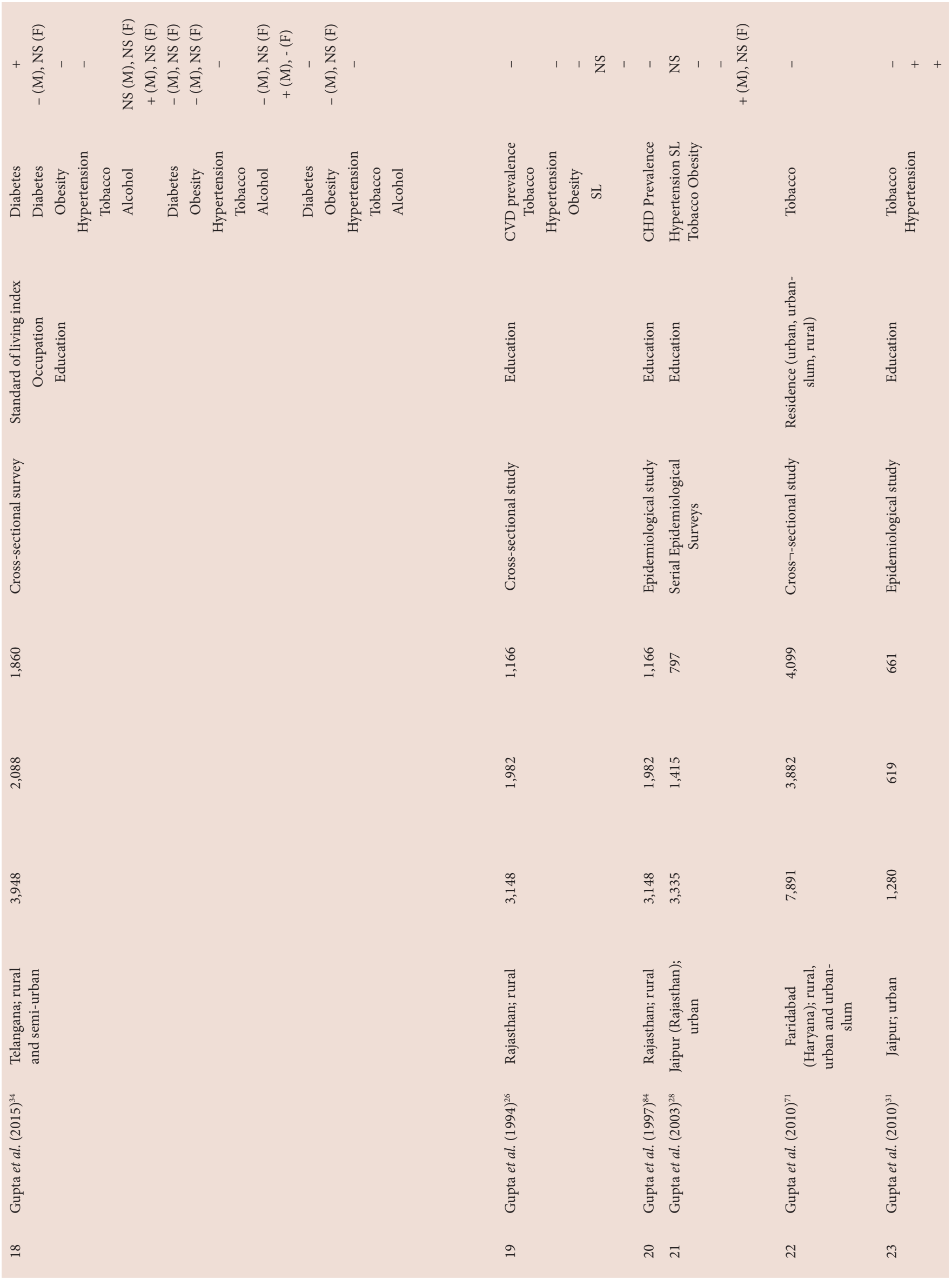




\begin{tabular}{|c|c|c|c|c|c|c|c|c|c|c|c|}
\hline $\begin{array}{llll}1 & 1 & 1 & 1\end{array}$ & 11 & $+\quad+$ & ++++ & 1 & 11 & $+\quad+$ & 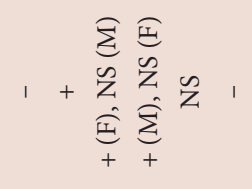 & + & $+\tilde{z}$ & 1 & $\tilde{z}+$ \\
\hline $\begin{array}{l}\overline{0} \\
\overline{0} \\
\frac{0}{4}\end{array}$ & 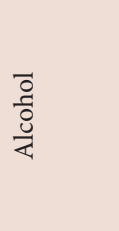 & 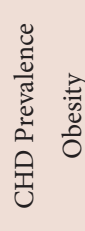 & 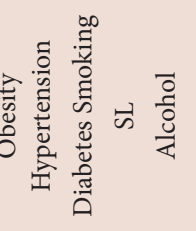 & 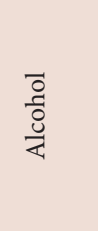 & $\ddot{\omega}$ & $\begin{array}{l}\text { 㐔 } \\
\text { ठัँ }\end{array}$ & 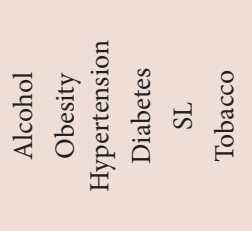 & 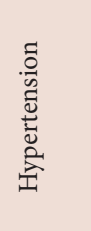 & 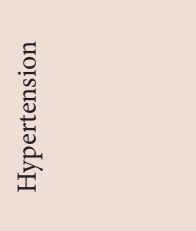 & 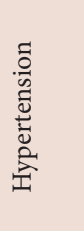 & 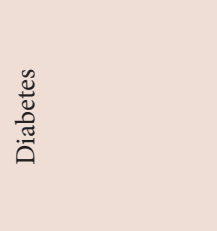 \\
\hline 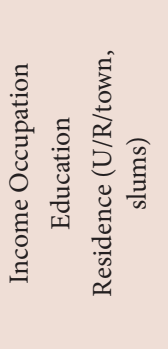 & 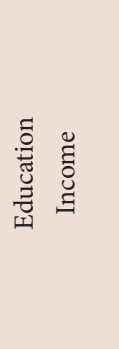 & 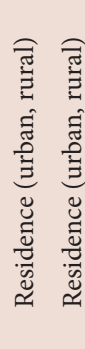 & & 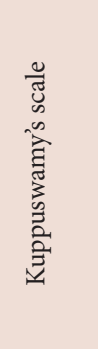 & 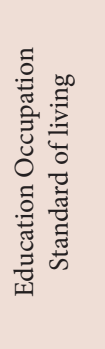 & 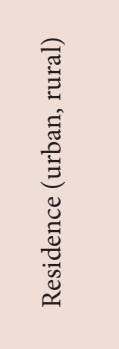 & 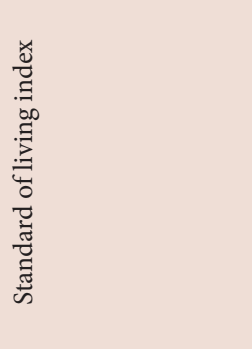 & 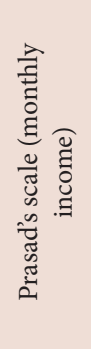 & 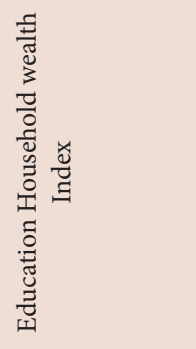 & 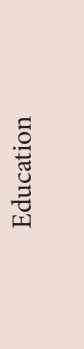 & 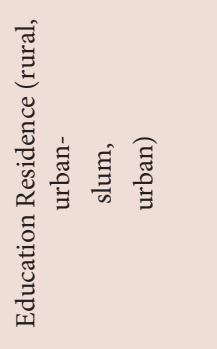 \\
\hline 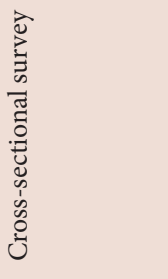 & 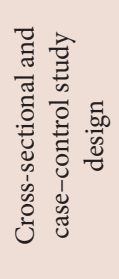 & 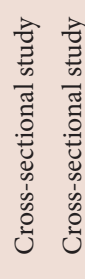 & 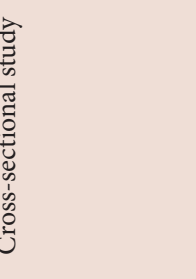 & 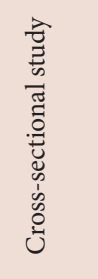 & 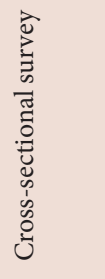 & 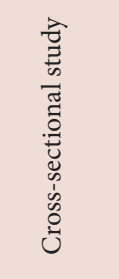 & 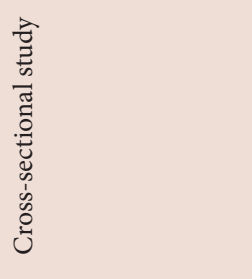 & 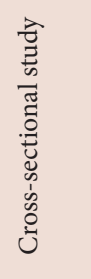 & 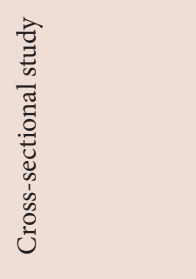 & 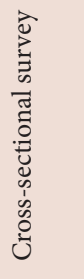 & 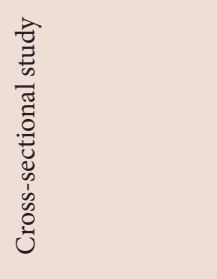 \\
\hline ֻ & | I & 乲 & I & 1 & 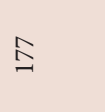 & 8 & $\frac{10}{6}$ & $\stackrel{\text { }}{\not}$ & $\begin{array}{l}8 \\
\text { i } \\
\text { î́ }\end{array}$ & $\vec{n}$ & 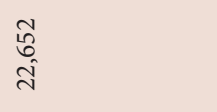 \\
\hline 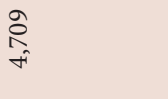 & 鬲 & 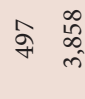 & $\begin{array}{l}\infty \\
\infty \\
\infty \\
\infty \\
m \\
m\end{array}$ & స్ల & $\stackrel{H}{\Xi}$ & 1 & $\stackrel{\infty}{\stackrel{0}{\sim}}$ & $\mathscr{Z}$ & $\begin{array}{l}\vec{\exists} \\
\vec{i}\end{array}$ & Ұ゙ & $\begin{array}{l}\infty \\
\infty \\
\rightarrow \vec{\sim} \\
\vec{\sim}\end{array}$ \\
\hline 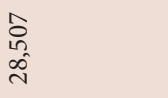 & 先 & $\begin{array}{c}\text { 왕 } \\
\text { : } \\
\text { - }\end{array}$ & 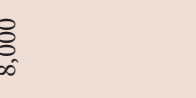 & ત્ત & $\vec{n}$ & 8 & $\stackrel{\infty}{\Omega}$ & নू & 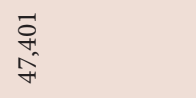 & $\stackrel{n}{\kappa}$ & $\begin{array}{l}\widetilde{N} \\
\text { N } \\
f\end{array}$ \\
\hline 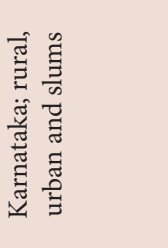 & 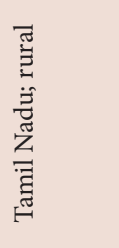 & 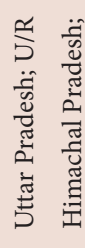 & 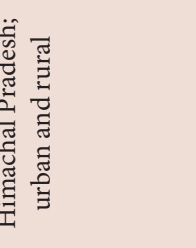 & 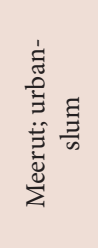 & 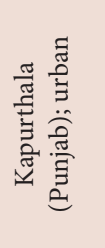 & 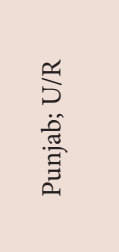 & 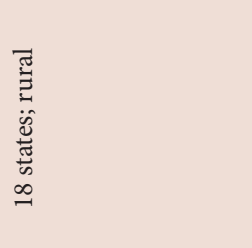 & 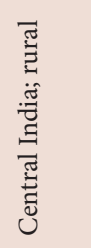 & 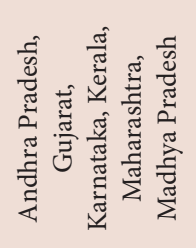 & 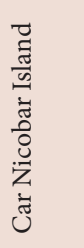 & 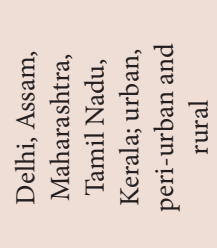 \\
\hline 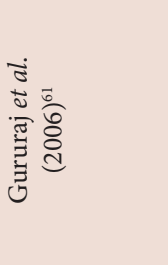 & 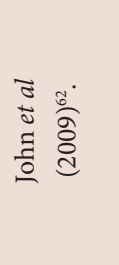 & 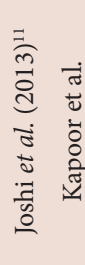 & 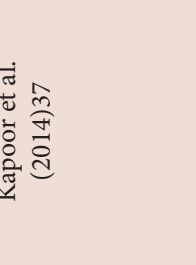 & 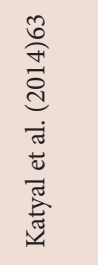 & 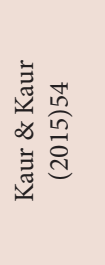 & 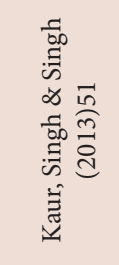 & 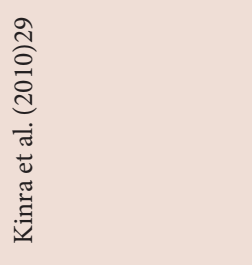 & 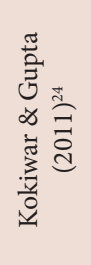 & 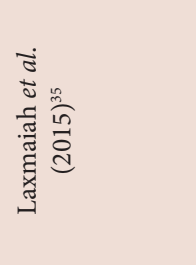 & 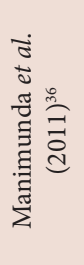 & 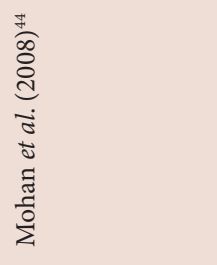 \\
\hline H & $\stackrel{\sim}{\sim}$ & $\stackrel{\sim}{\sim}$ ה & $\hat{~}$ & $\stackrel{\infty}{\sim}$ & $\stackrel{\infty}{\sim}$ & $\stackrel{m}{n}$ & $m$ & m & $m$ & $\ddot{m}$ & $\stackrel{n}{m}$ \\
\hline
\end{tabular}




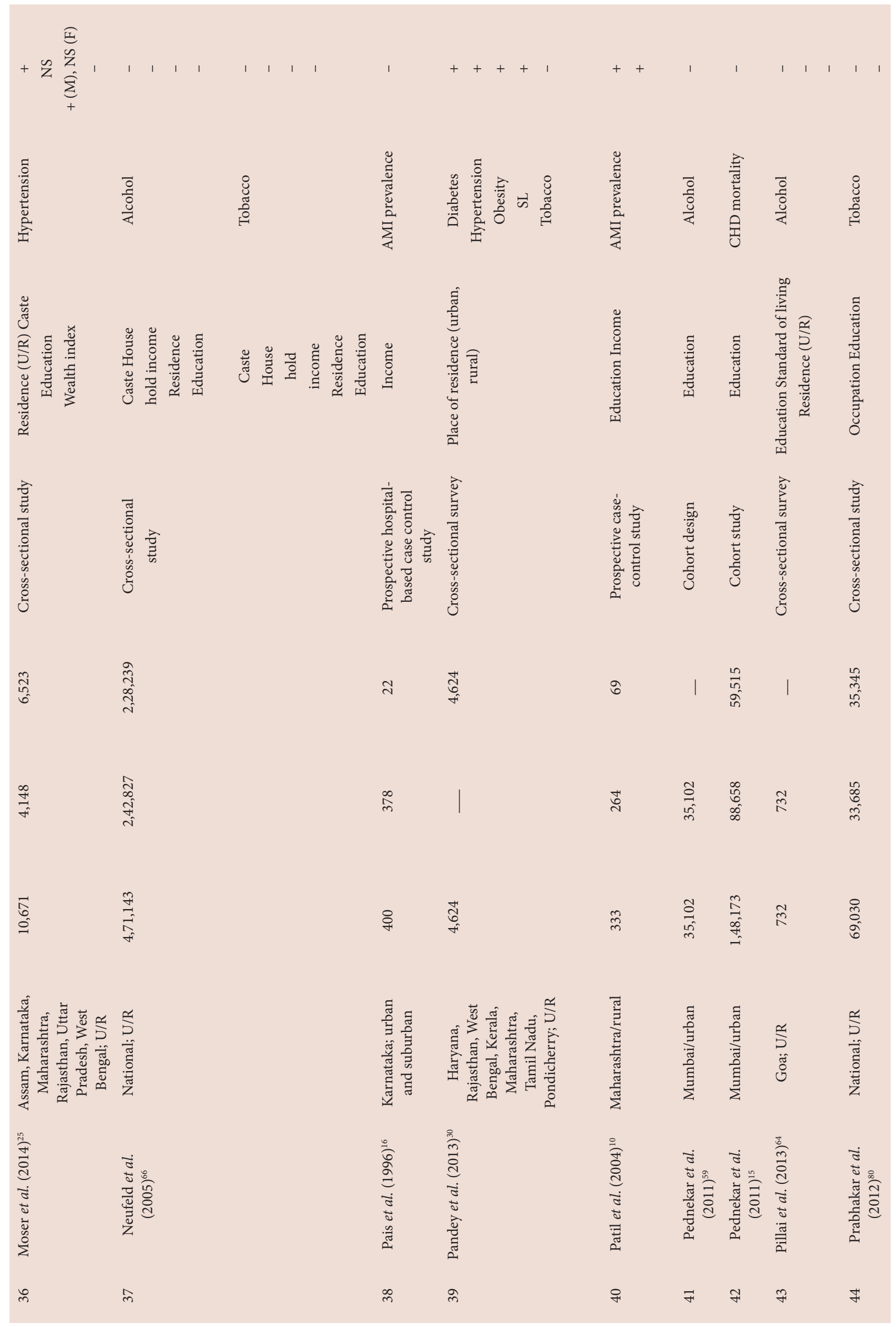




\begin{tabular}{|c|c|c|c|c|c|c|c|c|c|c|c|}
\hline+ & 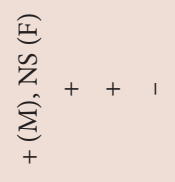 & $+\quad+$ & $\begin{array}{lll}1 & 1 & 1\end{array}$ & $+\quad++$ & + & & & ++ & 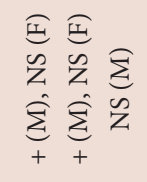 & 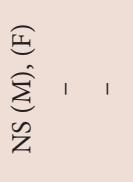 & 1 1 \\
\hline 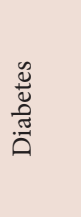 & 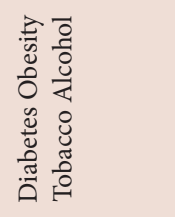 & 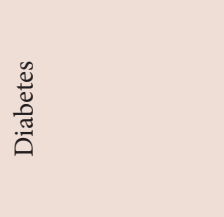 & 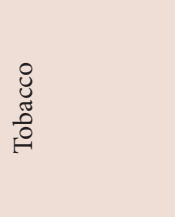 & 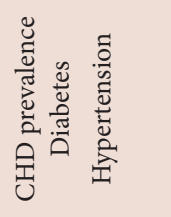 & 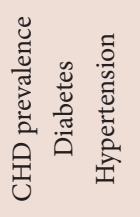 & & 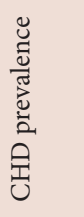 & $\begin{array}{l}\text { 㤹 } \\
\text { ठิ }\end{array}$ & 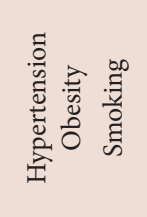 & 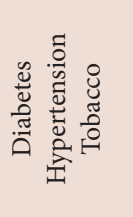 & $\begin{array}{l}\stackrel{8}{\mathscr{J}} \\
\stackrel{0}{0} \\
\stackrel{0}{\leftrightarrow}\end{array}$ \\
\hline 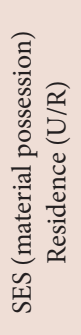 & & 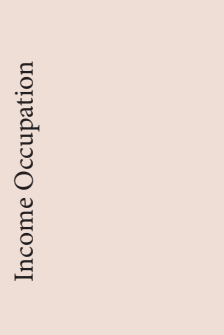 & 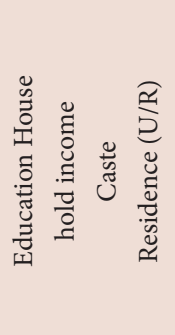 & 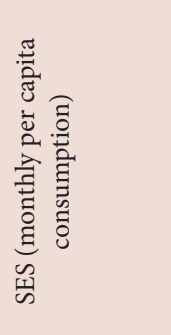 & & & 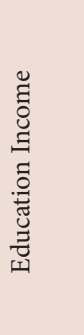 & 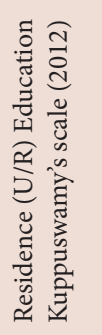 & 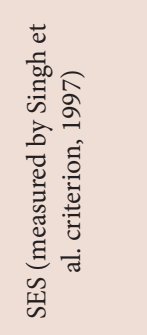 & 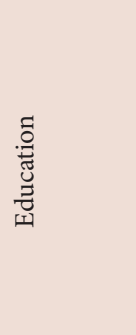 & 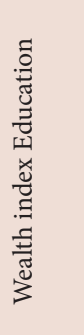 \\
\hline 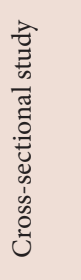 & & 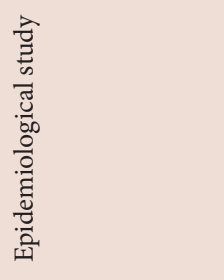 & 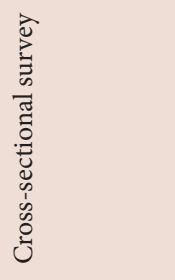 & 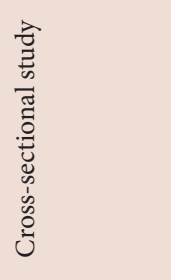 & & & 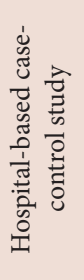 & 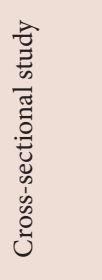 & 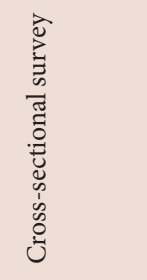 & 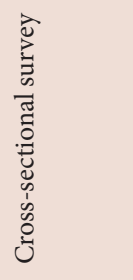 & 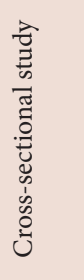 \\
\hline 岁 & & $\begin{array}{l}\infty \\
\text { ஸे }\end{array}$ & 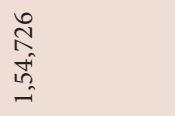 & $\stackrel{+}{=}$ & & & 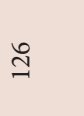 & $\tilde{\delta}_{-i}^{n}$ & $\stackrel{\circ}{\sim}$ & $\begin{array}{l}\hat{N} \\
\hat{0} \\
\infty\end{array}$ & I \\
\hline$\stackrel{\vec{\Xi}}{=}$ & & $\begin{array}{l}\infty \\
\infty \\
\text { in } \\
\text { in }\end{array}$ & $\begin{array}{l}\overrightarrow{1} \\
\infty \\
0 \\
0 \\
-1\end{array}$ & 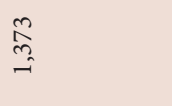 & & & নু & 1 & f & 网 & $\begin{array}{l}\hat{b} \\
\text { के } \\
\text { t }\end{array}$ \\
\hline 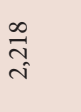 & & $\begin{array}{l}\stackrel{0}{N} \\
=\end{array}$ & $\begin{array}{l}\infty \\
\text { on } \\
\text { n̂े } \\
\text { nी }\end{array}$ & 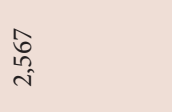 & & & 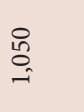 & $\tilde{\delta}_{-i}^{0}$ & 웅 & $\begin{array}{l}\tilde{a} \\
\hat{\sigma}\end{array}$ & 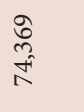 \\
\hline 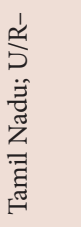 & & 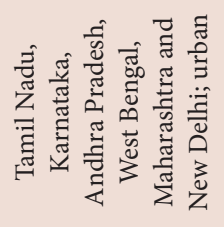 & 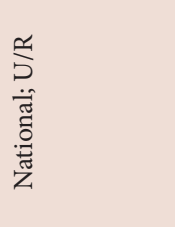 & 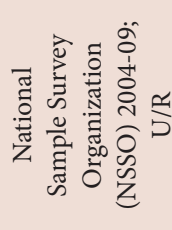 & & & 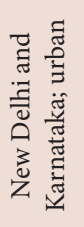 & 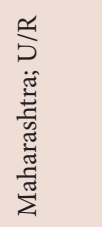 & 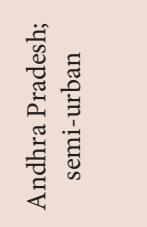 & 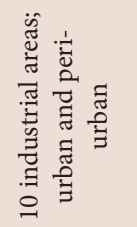 & 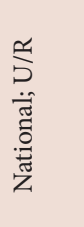 \\
\hline 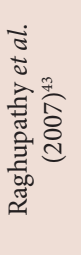 & & 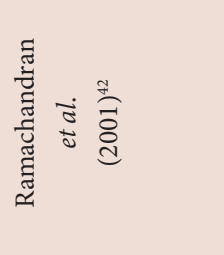 & 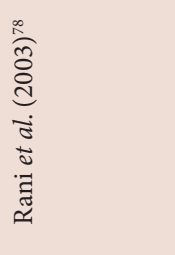 & 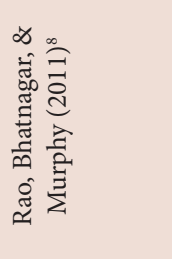 & & & 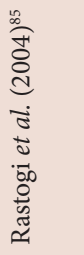 & 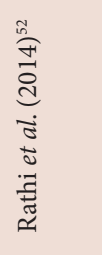 & 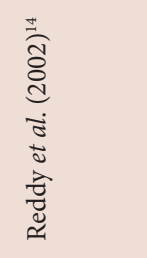 & 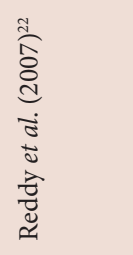 & 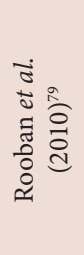 \\
\hline \& & & fo & f & $\stackrel{\infty}{\rightarrow}$ & & & भे & in & $\vec{n}$ & in & in \\
\hline
\end{tabular}




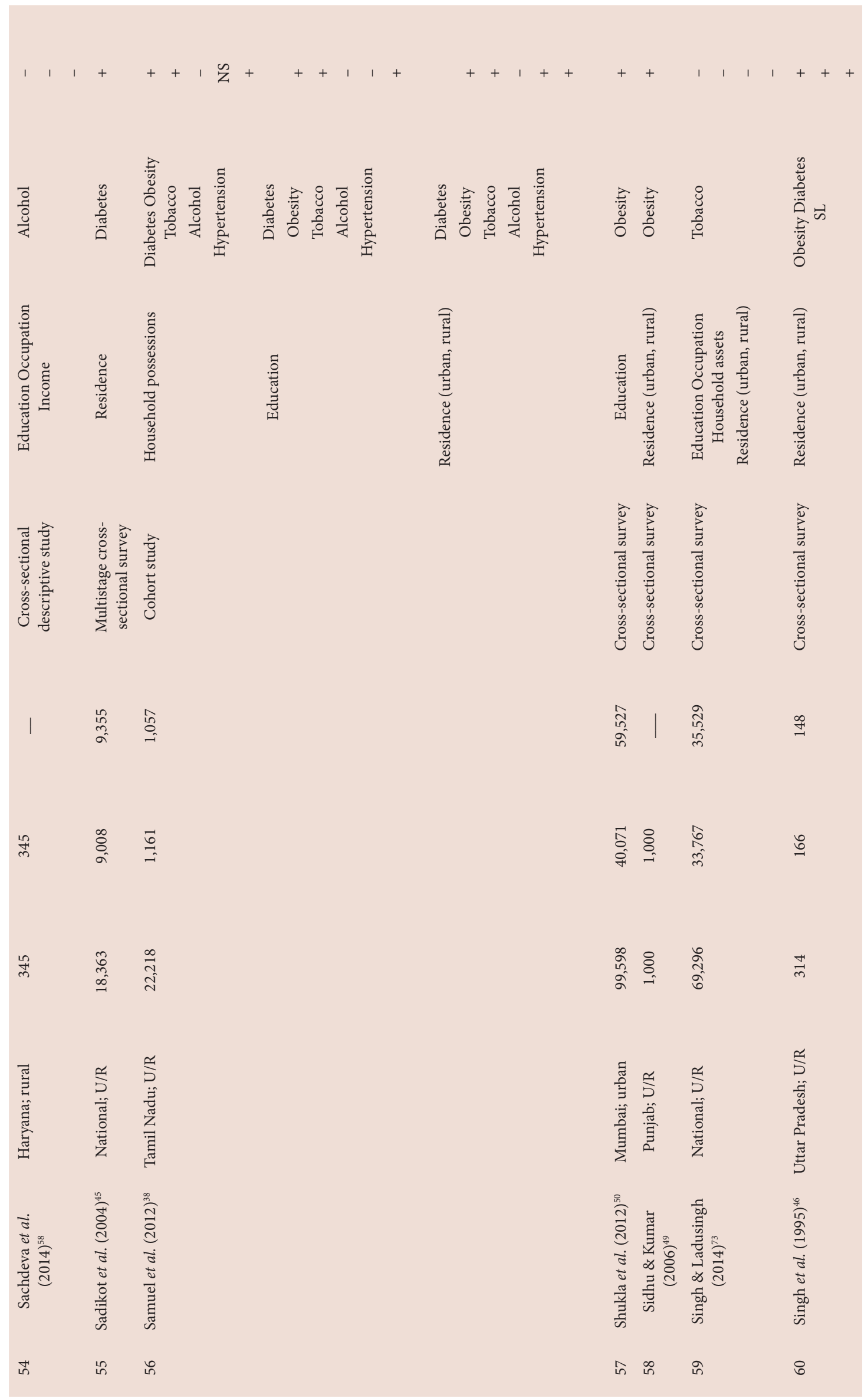




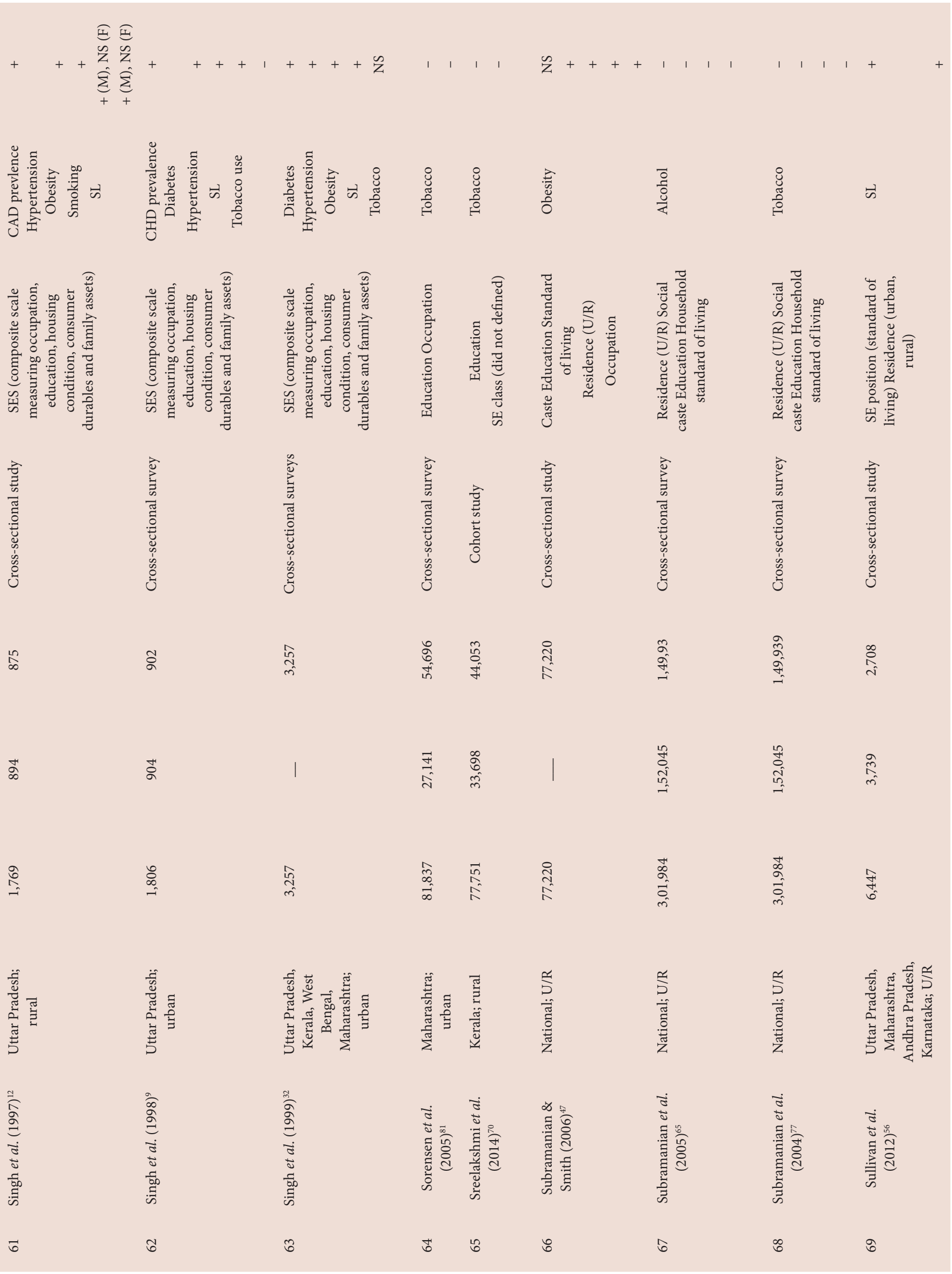




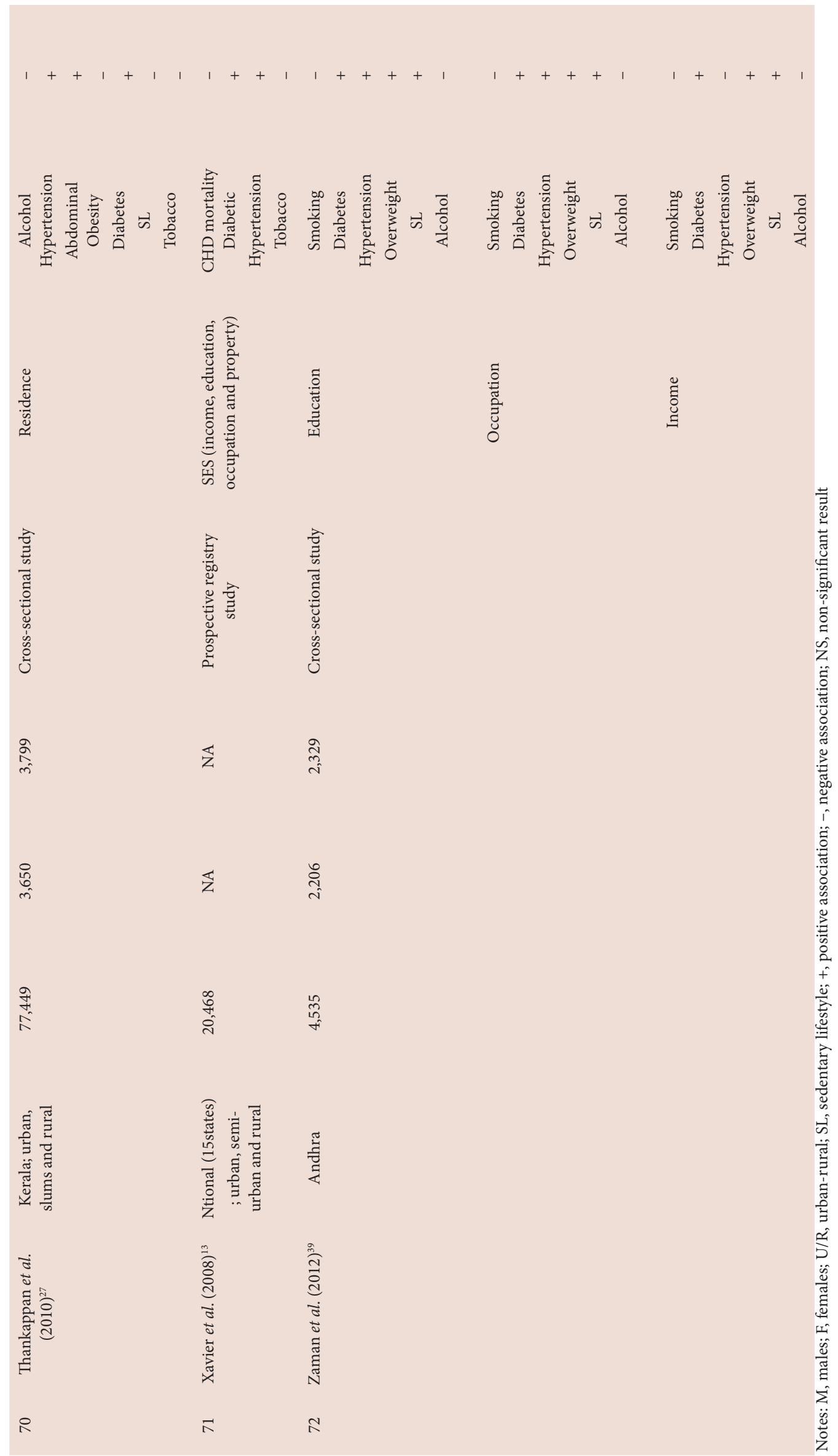




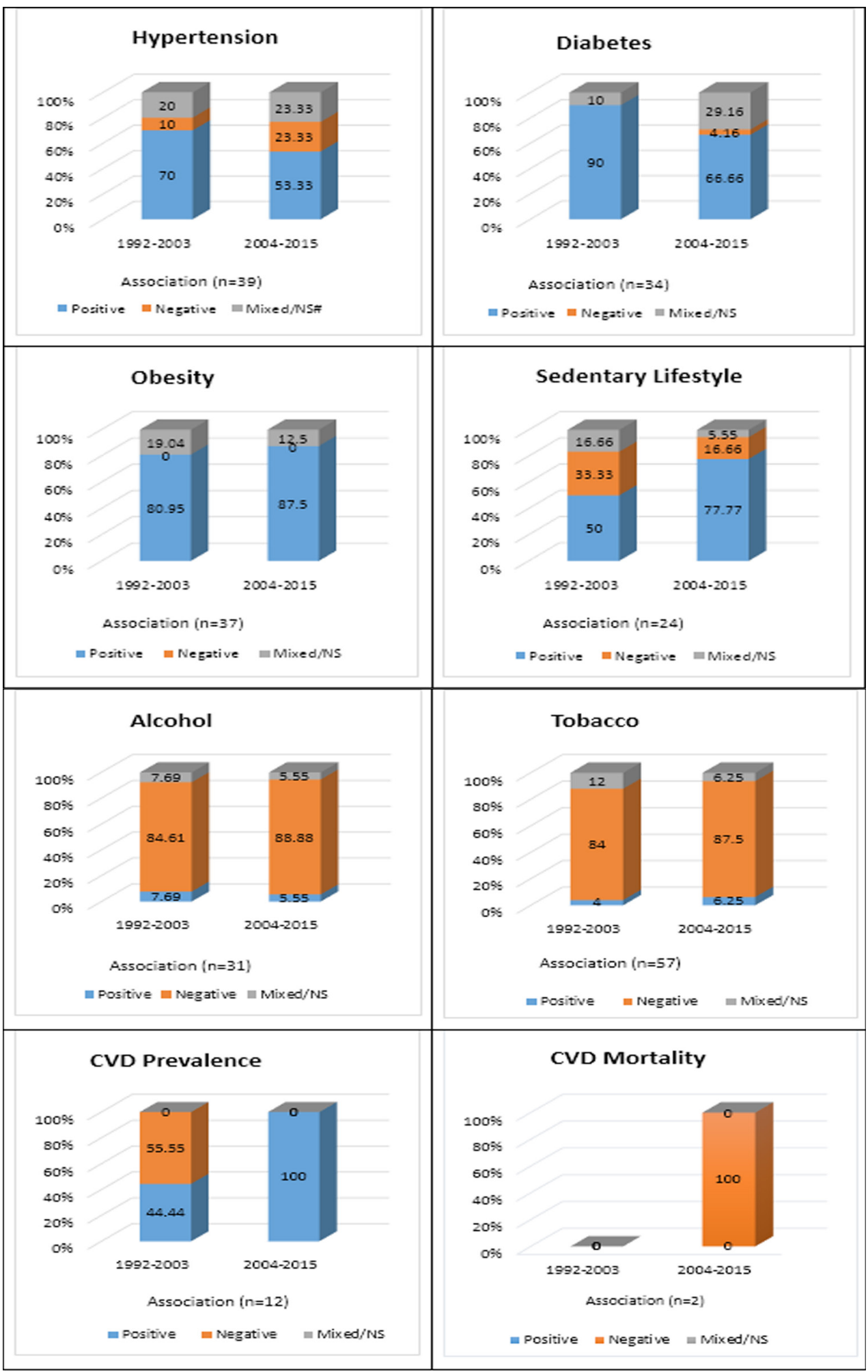

Figure 3: Direction of association (positive, negative, mixed/non-significant) between cardiovascular risk factors, prevalence and mortality w.r.t. socioeconomic status, stratified based on the year of data collection (1992-2003 and 2004-2015) from 72 studies and 236 associations. \# Mixed association or non-significant (NS) association refers to different results reported either w.r.t. male and female participants or w.r.t urban and rural participants in the same study

\section{Obesity}

All 21 selected studies ${ }^{12,14,26-32,34,37,38,39,43,46,47,49-53}$ have either used the general criteria of WHO for obesity (BMI $\geq 30$ or $\geq 25)$ or South-Asia-specific criteria (BMI $\geq 23$ ). Except for four non-significant and mixed result associations, all the studied associations w.r.t. education, income, residence and occupation have reported a positive association between the SES and 
obesity (Figure 2). Again, unlike developed countries, in India people from higher SES are more prone to obesity. Four studies have been carried out on female participants only ${ }^{30,32,47,51}$ and they report similar results as those conducted on male participants, suggesting that SES affects both genders in the same way.

\section{Sedentary lifestyle}

Sixteen studies ${ }^{9,14,22,23,26,27-30,32,37,39,54-57}$ were reviewed to determine the effect of sedentary lifestyle on CVD. In these studies, varied criteria were used for measuring sedentary lifestyle, including Johnson Space Centre (JSC) physical activity scale, ${ }^{51}$ MET scores calculated using WHO's Global Physical Activity Questionnaire ${ }^{43,53}$ Paffenberger's criteria ${ }^{23}$ and the scale developed by Bharthi et al. ${ }^{26,27}$ Out of the 18 studied associations w.r.t. education, income, residence and occupation, 13 reported positive and 5 reported negative relationships between sedentary lifestyle and SES (Figure 2). Thus, these studies support the view that people belonging to higher SES are more physically inactive than their lower SES counterparts.

\section{Alcohol}

All 13 studies s7,29,34,58-67 $^{2}$ selected to study the effect of alcohol reported a negative relationship of alcohol intake with SES. Of these 13 studies, seven were done only on male participants. These studies investigated 27 associations w.r.t. four SES markers; except four, all reported a negative relationship between the SES and alcohol intake (Figure 2). These studies have either used the AUDIT (Alcohol Use Disorder Identification Test), questionnaire to report the prevalence rate or some other self-reporting method. Lower strata tend to use country liquor more and they are the most frequent and heavy drinkers..$^{59,68}$ Caste, education and standard of living have also been reported to affect the alcohol consumption behaviour. ${ }^{65}$ In the same study, it has been reported that the relationship between education and alcohol consumption is different among men and women; while men show an inverse relationship, a U-shaped relation has been reported for women. ${ }^{65}$

\section{Tobacco}

Thirty-one studies ${ }^{9,12,13,22,26,28-31,34,38,55,66,69-83}$ were identified to investigate the effect of tobacco on CVD. All these studies investigated the association of the four most used SES markers and reported a negative association with tobacco use (Figure 2). These studies considered tobacco consumption either in smoke or/and smokeless form. Use of smokeless tobacco (26\%) has been found to be more prevalent than smoking (14\%) ${ }^{69}$ Bidi (a form of cigarette) is used commonly in rural and urban areas by poor section across India. ${ }^{83}$ Irrespective of the urban and rural setup, lower SES groups consume more tobacco, although the type of tobacco differs. With increase in education, all kinds of tobacco usage declined except for cigarette usage by men. ${ }^{69,76,81}$ High usage of tobacco has been reported in self-employed participants and non-government employees of both genders, and it was highest in those participants who were unemployed but were capable of working. Student community reported the lowest use of tobacco. ${ }^{81}$

\section{Markers of socio-economic status}

Among the studies we selected, 39 have used the place of residence as a marker in the form of a U/R difference; some studies have also included semi-urban and urban-slum areas along with U/R differences. Residence as a socioeconomic marker reported a negative association for all CVRFs, except for alcohol. Thus, these studies suggest a clear U/R division in India, where urban residents are positively associated with all CVRFs, except for alcohol. Interestingly, out of six reported associations between alcohol and residence, two were positive, three were negative and one was a mixed association, suggesting that both urban and rural populations are affected by alcohol.

Another important SES marker of CVD is education and it is also the most studied socioeconomic marker; 52 studies reviewed in this paper employed it. In our analysis, however, education consistently predicted negative associations with alcohol and tobacco throughout the timeline of our systematic review, but it produced a highly variable pattern of association with hypertension, followed by the remaining three CVRFs (diabetes, sedentary lifestyle and obesity). This suggests that urban population belonging to higher educated strata is more vulnerable to hypertension, diabetes, sedentary lifestyle and obesity, but not to alcohol and tobacco consumption. An important difference also exists in the context of tobacco consumption between urban and rural residents (as discussed earlier in the Tobacco section). Moreover, another review on Asian Indians has reported that urban Indian population is more susceptible to CVD and its risk factors than the rural population of India. ${ }^{84}$

\section{CVD across Socioeconomic Status}

No consensus has been found among the 11 studies $^{8-13,15,26,85-87}$ that have been identified to understand the trend of CVD w.r.t. SES. Five studies ${ }^{8-12}$ have reported a positive association, while the other $\operatorname{six}^{13,16,26,85-87}$ reported a negative association between the CVD and SES of participants. Two of these 11 studies ${ }^{13,15}$ have been analysed for understanding the pattern of CVD mortality across SES. Even though both studies have used different markers of SES, they reported a negative relationship between the SES and CVD mortality. Both of them reported a higher mortality rate among lower SES strata and least educated people. Lower SES patients were also found to receive least evidence-based treatment. ${ }^{13}$ These studies $^{13,15}$ suggest that even though there is no clear pattern of association between the CVD and SES, the mortality is clearly higher among lower SES groups.

\section{Socioeconomic Patterning of CVD And Its Risk Factors Across Time}

Total 236 associations from 72 studies were extracted and stratified w.r.t. to timeline (1992-2003 and 2004-2015) to understand the changing pattern of CVD and its risk factors in the last 25 years. This division of timeline was done to create equal time-intervals. The results showed that the percentage of negative association for hypertension and diabetes has increased over time, suggesting an increase in the number of hypertensive and diabetic patients from lower SES groups (Figure 3). In contrast, percentage of positive association has increased for sedentary lifestyle and obesity, i.e. the percentage of people from higher SES groups having sedentary lifestyle and more obesity has increased in the last 25 years. However, we could not find any significant change in the alcohol and tobacco consumption pattern w.r.t. SES, i.e. lower SES sections still bear the majority of its burden. All studies conducted during 2004-2015 have reported a positive association w.r.t. CVD prevalence in comparison to the earlier timeline of 1992-2003, but the burden of its mortality can be seen to lie completely with the lower SES section.

\section{DISCUSSION}

This systematic review suggests that the pattern of CVD in India differs from that of developed countries, where both CVD prevalence and its risk factors are associated with lower SES. Our systematic review shows that although in India there is no clear pattern of association between CVD and SES, the same is not true with the association between CVRFs and SES. Based on the results, we can conclude that only two risk factors (tobacco and alcohol consumption) out of six that were studied are linked to lower SES, but the mortality rate due to CVD is higher among lower SES groups. Similar results were also reported in another review, ${ }^{17}$ 
which concluded that except smoking and low fruit and vegetable intake, other CVRFs are more prevalent in higher SES strata. Our systematic review reported a mixed result of CVD prevalence across SES, which could be the manifestation of the ongoing epidemiological transition in India. Moreover, as there is no consensus among studies over the SES patterning of CVD, it is too early to suggest a reversal of the social gradient and one should be cautious about proposing any such conclusion. With economic liberalisation, development and globalisation, the relationship between the CVD and SES is changing; however, it has not yet reached that point in India from where a reversal in the social gradient can occur. Because of this on-going process of development, one can see many, but not all, studies reporting a reversal of social gradient. Moreover, according to Ezzati et al.$^{88}$ when a nation's GNI (gross national income) crosses US\$ 5000, only then there will be a reversal in the relationship between the SES and risk factors of a specific disease. Data from the World Bank show that India's GNI has not crossed the US\$ 5000 mark yet; ${ }^{88}$ thus, we can expect to have a negative relationship between the SES and the prevalence of risk factors and mortality in near future but not now.

A higher mortality among lower SES strata does not indicate the reversal of the social gradient in the context of CVD; instead, it indicates the occurrence of other important factors such as lesser accessibility to treatment facilities by lower SES groups, ${ }^{13}$ lower level of education and its negative impact on health-related behaviour, ${ }^{22,90}$ poor government policies ${ }^{65}$ and malnutrition in lower SES strata. ${ }^{91}$ In a landmark study, ${ }^{13}$ Xavier et al. reported that patients belonging to lower SES groups receive less evidence-based treatment and a lesser interventional treatment. Only $9 \%$ patients cover their cost of treatment either through insurance, employer or through government funding. They also reported that patients of lower SES were not able to afford secondary prevention treatment and routine treatments in hospitals. Inability of patients to reach hospital in time is another factor that increases the mortality rate. Due to lack of good ambulance services, traffic problems and longer distance from hospitals, patients reach the hospital quite late and sometimes they die before reaching there. These factors affect weaker sections and rural people more as they do not own any private vehicle and rural areas have generally poor ambulance services. Xavier et al. also proposed that with equal accessibility of treatment facilities, mortality difference across different SES strata could disappear.

Loopholes in government policies can influence the mortality and morbidity rates of CVD as well as the presence or absence of its risk factors. In particular, higher prevalence of alcohol and tobacco use in lower SES people can be attributed to government policies. In India, it is the responsibility of the state to make policies on alcohol production and consumption..$^{65}$ As a result, states have different alcohol consumption rates. Many studies ${ }^{92,93}$ have reported that because of its easy availability and low price, poor people tend to use country liquor more, ${ }^{59}$ which is sometimes adulterated with a high level of ethanol. However, there are still no strict rules or policies for monitoring country liquor in India. As rightly put, 'The politics of alcohol in India is complex', as both the Indian government and Indian economy depend on alcohol industry for revenue and generation of jobs; therefore, it is difficult for the government to put stringent laws to control alcohol production and consumption. ${ }^{62}$

The government should also impart a rural orientation in all its tobaccocontrol programs as all strata of the rural population are equally affected by tobacco use. ${ }^{70}$ Bidi smoking is also a common form of tobacco use in rural area. As per government policies, bidi manufacturing industry is considered a small-scale industry. Hence, many policies support the bidi industry..$^{90}$ Another reason for the higher prevalence of CVD in lower SES groups could be malnutrition. Though they are more physically active because of their occupation-related requirements, this does not give them an advantage against CVD because of their poor diet. ${ }^{26}$

\section{CONCLUSION AND RECOMMENDATIONS}

The following findings can be summarised from the present review:

- CVD risk factors are more associated with higher SES, but lower SES groups have higher mortality.

- Mixed results on CVD patterning and risk factors suggest that the Indian society is undergoing an epidemiological transition.

- $\quad$ Education, as a marker of SES, plays an important role in determining the health behaviour.

- Urban population with higher SES is more prone to CVD prevalence and CVRFs (hypertension, diabetes, obesity and sedentary lifestyle), while that with lower SES is more prone to CVD mortality. In contrast, both urban and rural populations with lower SES have a higher percentage of tobacco and alcohol consumption than their counterparts with higher SES.

- Factors such as treatment facilities and government policies play an important role in affecting CVD and its associated risk factors.

The majority of reviewed studies have used education as a SES marker and found its significant role in predicting the risk factors. As education decreases, it decreases the awareness on the ill effects of smoking, alcohol, diet, physical exercise and healthy behaviours and also negatively influences the adherence behaviour. ${ }^{93}$ As lower strata have less financial resources and cannot afford expensive CVD treatments, self-management practices can play an important part in lowering down their mortality rate. The government should take an active role in organising CVD rehabilitation programs, especially for weaker sections, so that they can be made aware about self-management practices and various risk factors and health issues related to CVD.

Policy makers should take into account the patterning of CVRFs while formulating policies and programs to curb CVD. In India, formulating policies for CVD will be a difficult challenge for policy makers, because although higher SES strata are suffering more from CVRFs, lower SES strata have a higher CVD mortality rate. If India wants to control the CVD epidemic, then its policy makers need to take into account both strata. Special attention should be given to factors responsible for unequal distribution of medical facilities among different SES strata. Special attention should also be given to the problem of higher tobacco and alcohol consumption among lower SES groups as it can lead to health problems other than CVD.

\section{ACKNOWLEDGEMENT}

None

\section{CONFLICT OF INTEREST}

None

\section{REFERENCES}

1. World Health Organization. Diet, nutrition and the prevention of chronic diseases. World Health Organ Tech Rep Ser 2003;916. [Cited 2014 December 24] Avaliable from: http://www.who.int/dietphysicalactivity/publications/trs916/ download/en/

2. UNDP. About India. [Internet] [Cited 2015 Januray 30] Avaliable from: http:// www.in.undp.org/content/india/en/home/countryinfo/.

3. Mackay J, Mensah GA, Mendis S, Greenlund K. The atlas of heart disease and stroke. World Health Organization; 2004.

4. Kaplan GA, Keil JE. Socioeconomic Factors and Cardiovascular Disease : A review of the literature. Circulation 1993;88:1973-98. http://dx.doi.org/10.1161/01. CIR.88.4.1973.

5. Reddy KS, Yusuf S. Emerging Epidemic of Cardiovascular Disease in Developing Countries. Circulation 1998;97:597-601. http://dx.doi.org/10.1161/01 CIR.97.6.596.

6. Jeemon P Reddy KS. Social determinants of cardiovascular disease outcomes in Indians. Indian J Med Res 2010;132:617-22. PMid:21150014 
Singh et al.: Socio-economic Patterning of Cardiovascular Disease and its Risk Factors among Indians

PMCid:PMC3028951.

7. Sarvotham SG, Berry JN. Prevalence of Coronary Heart Disease in an Ur ban Population in Northern India. Circulation 1968;37:939-53. http://dx.doi. org/10.1161/01.CIR.37.6.939

8. Rao KD, Bhatnagar A, Murphy A. Socio-economic inequalities in the financing of cardiovascular \& diabetes inpatient treatment in India. Indian J Med Res 2011;133:57-63. PMid:21321420 PMCid:PMC3100147.

9. Singh RB, Sharma JP, Rastogi V, Niaz MA, Ghosh S, Beegom R, et al. Social class and coronary artery disease in a urban population of North India in the Indian lifestyle and heart study. Eur Heart J 1998:64:195-203. http://dx.doi. org/10.1016/s0167-5273(98)00048-5.

10. Patil SS, Joshi R, Gupta G, Reddy MVR, Pai M, Kalantri SP. Risk factors for acute myocardial infarction in a rural population of central India: a hospital-based case-control study. Natl Med J India 2004;17:189-94. PMid:15372760.

11. Joshi AV, Hungund BR, Katti SM, Viveki RG. Prevalence of hypertension and its socio demographic and occupational determinants among bus drivers in North Karnataka - A Cross sectional study 2013;2:3-7.

12. Singh RB, Sharma JP, Rastogi $V$, Niaz MA, Ghosh S, Beegom R, et al. Social class and coronary disease in rural population of north India. The Indian Social Class and Heart Survey. Eur Heart J 1997;18:588-95. http://dx.doi.org/10.1093/ oxfordjournals.eurheartj.a015301; PMid:9129887.

13. Xavier D, Pais P, Devereaux PJ, Xie C, Prabhakaran D, Reddy KS, et al. Treatment and outcomes of acute coronary syndromes in India (CREATE): a prospective analysis of registry data. Lancet 2008;371:1435-42. http://dx.doi.org/10.1016/ S0140-6736(08)60623-6

14. Reddy KKR, Rao AP, Reddy TPK. Socioeconomic status and the prevalence of coronary heart disease risk factors. Asia Pac J Clin Nutr 2002;11:98-103. http:// dx.doi.org/10.1046/j.1440-6047.2002.00267.x ; PMid:12074188.

15. Pednekar MS, Gupta R, Gupta PC. Illiteracy, low educational status, and cardiovascular mortality in India. BMC Public Health 2011;11:567. http://dx.doi. org/10.1186/1471-2458-11-567 ; PMid:21756367 PMCid:PMC3160988.

16. Pais P, Pogue J, Gerstein H, Zachariah E, Savitha D, Jayprakash S, et al. Risk factors for acute myocardial infarction in Indians: a case-control study. Lancet 1996;348:358-63. http://dx.doi.org/10.1016/S0140-6736(96)02507-X.

17. Subramanian SV., Corsi DJ, Subramanyam MA., Smith GD. Jumping the gun: The problematic discourse on socioeconomic status and cardiovascular health in India. Int J Epidemiol 2013;42:1410-26. http://dx.doi.org/10.1093/ije/dyt017 ; PMid:23563358.

18. Jones-Smith JC. Commentary: Jumping the gun or asleep at the switch: is there a middle ground? Int J Epidemiol 2013;42:1435-7. http://dx.doi.org/10.1093/ije/ dyt080 ; PMid:24008331 PMCid:PMC3807612.

19. Subramanian SV., Subramanyam MA., Corsi DJ, Smith GD. Rejoinder: Need for a data-driven discussion on the socioeconomic patterning of cardiovascula health in India. Int J Epidemiol 2013;42:1438-43. http://dx.doi.org/10.1093/ije/ dyt181; PMid:24019422.

20. Kurian NJ. Widening economic \& social disparities: Implications for India. Indian J Med Res 2007;126:374-80. PMid:18032812.

21. Hnatkovska V, Lahiri A. The rural-urban divide in India. Int Growth Centr Work Pap. 2013.

22. Reddy KS, Prabhakaran D, Jeemon P, Thankappan KR, Joshi P, Chaturvedi V, et al. Educational status and cardiovascular risk profile in Indians. Proc Natl Acad Sci U S A 2007;104:16263-8. doi:10.1073/pnas.0700933104. http://dx.doi. org/10.1073/pnas.0700933104.

23. Bhadoria AS, Kasar PK, Toppo N a, Bhadoria P, Pradhan S, Kabirpanthi V. Prevalence of hypertension and associated cardiovascular risk factors in Central India. J Family Community Med 2014:21:29-38. http://dx.doi.org/10.4103/22308229.128775; PMid:24695988 PMCid:PMC3966094.

24. Kokiwar PR, Gupta SS, Durge PM. Prevalence of hypertension in a rural community of central India. J Assoc Physicians India. 2012;60:26-29. PMid:23409417.

25. Moser KA, Agrawal S, Davey Smith G, Ebrahim S. Socio-demographic inequalities in the prevalence, diagnosis and management of hypertension in India: analysis of nationally-representative survey data. PLoS One 2014;9:e86043. http://dx.doi.org/10.1371/journal.pone.0086043

PMid:24465859 PMCid:PMC3900470

26. Gupta R, Gupta VP, Ahluwalia NS. Educational status, coronary heart disease, and coronary risk factor prevalence in a rural population of India. BMJ 1994;309:1332-6. http://dx.doi.org/10.1136/bmj.309.6965.1332 ; PMid:7866081 PMCid:PMC2541843.

27. Thankappan KR, Shah B, Mathur P, Sarma PS, Srinivas G, Mini GK, et al. Risk factor profile for chronic non-communicable diseases: Results of a communitybased study in Kerala, India. Indian J Med Res 2010;131:53-63. PMid:20167974.

28. Gupta R, Gupta VP, Sarna M, Prakash H, Rastogi S, Gupta KD. Serial epidemiological surveys in an urban Indian population demonstrate increasing coronary risk factors among the lower socioeconomic strata. J Assoc Physicians India 2003;51:470-7. PMid:12974428.

29. Kinra S, Bowen LJ, Lyngdoh T, Prabhakaran D, Reddy KS, Ramakrishnan L, et al. Sociodemographic patterning of non-communicable disease risk factors in rura India: a cross sectional study. BMJ 2010;341:c4974. http://dx.doi.org/10.1136/ bmj.c4974 ; PMid:20876148 PMCid:PMC2946988.
30. Pandey RM, Gupta R, Misra A, Misra P, Singh V, Agrawal A, et al. Determinants of urban-rural differences in cardiovascular risk factors in middle-aged women in India: A cross-sectional study. Int J Cardiol 2013:163:157-62. http://dx.doi. org/10.1016/j.ijcard.2011.06.008; PMid:21880382.

31. Gupta R, Kaul V, Agrawal A, Guptha S, Gupta VP. Cardiovascular risk according to educational status in India. Prev Med (Baltim) 2010;51:408-11. http://dx.doi. org/10.1016/j.ypmed.2010.08.014 PMid:20817021.

32. Singh RB, Beegom R, Mehta AS, Niaz M A, De AK, Mitra RK, et al. Social class, coronary risk factors and undernutrition, a double burden of diseases, in women during transition, in five Indian cities. Int J Cardiol 1999;69:139-47. http://dx.doi.org/10.1016/S0167-5273(99)00010-8.

33. Farag YM, Mittal B V, Keithi-Reddy S, Acharya VN, Almeida AF, C Anil, et al. Burden and predictors of hypertension in India: results of SEEK (Screening and Early Evaluation of Kidney Disease) study. BMC Nephrol 2014:15:42. http:// dx.doi.org/10.1186/1471-2369-15-42 ; PMid:24602391 PMCid:PMC4015417.

34. Gupta V, Millett C, Walia GK, Kinra S, Aggarwal A, Prabhakaran P, et al. Socioeconomic patterning of cardiometabolic risk factors in rural and peri-urban India: Andhra Pradesh children and parents study (APCAPS). J Public Health (Bangkok) 2015;23:129-36. http://dx.doi.org/10.1007/s10389-015-0662-y ; PMid:26000232 PMCid:PMC4434856

35. Laxmaiah A, Meshram II, Arlappa N, Balakrishna N, Rao KM, Reddy CG, et al. Socio-economic \& demographic determinants of hypertension \& knowledge, practices \& risk behaviour of tribals in India. Indian J Med Res 2015;141:697708. PMid:26139790 PMCid:PMC4510771.

36. Manimunda SP, Sugunan AP, Benegal V, Balakrishna N, Rao MV, Pesala KS. Association of hypertension with risk factors \& hypertension related behaviour among the aboriginal Nicobarese tribe living in Car Nicobar Island, India. Indian J Med Res 2011;133:287-93. PMid:21441682 PMCid:PMC3103153.

37. Kapoor D, Bhardwaj AK, Kumar D, Raina SK. Prevalence of Diabetes Mellitus and Its Risk Factors among Permanently Settled Tribal Individuals in Tribal and Urban Areas in Northern State of Sub-Himalayan Region of India. Int J Chronic Dis 2014;2014:9. http://dx.doi.org/10.1155/2014/380597.

38. Samuel P, Antonisamy B, Raghupathy P, Richard J, Fall CH. Socio-economic status and cardiovascular risk factors in rural and urban areas of Vellore, Tamilnadu, South India. IntJEpidemiol 2012;41:1315-27. http://dx.doi.org/10.1093/ ije/dys001.

39. Justin Zaman M, Patel A, Jan S, Hillis GS, Raju PK, Neal B, et al. Socio-economic distribution of cardiovascular risk factors and knowledge in rural India. Int J Epidemiol 2012;41:1302-14. http://dx.doi.org/10.1093/ije/dyr226 ; PMid:22345313.

40. Agrawal S, Ebrahim S. Prevalence and risk factors for self-reported diabetes among adult men and women in India: findings from a national cross-sectional survey. Public Health Nutrition. 2012;15(06):1065-77. http://dx.doi.org/10.1017/ S1368980011002813; PMid:22050916 PMCid:PMC3458429.

41. Corsi DJ, Subramanian S V. Association between socioeconomic status and self-reported diabetes in India: a cross-sectional multilevel analysis. BMJ Open 2012;2:e000895-e000895. http://dx.doi.org/10.1136/bmjopen-2012-000895 PMid:22815470 PMCid:PMC3401832.

42. Ramachandran A, Snehalatha C, Kapur A, Vijay V, Mohan V, Das AK, et al. High prevalence of diabetes and impaired glucose tolerance in India: National Urban Diabetes Survey. Diabetologia 2001;44:1094-101. http://dx.doi.org/10.1007/ s001250100627; PMid:11596662

43. Raghupathy P, Antonisamy B, Fall CHD, Geethanjali FS. High prevalence of glucose intolerance even among young adults in south India 2007;77:269-79.

44. Mohan V, Sandeep S, Deepa R, Shah B, Varghese C. Epidemiology of type 2 diabetes: Indian scenario. Indian J Med Res 2007;125:217-30. PMid:17496352.

45. Sadikot SM, Nigam A, Das S, Bajaj S, Zargar AH, Prasannakumar KM, et al. The burden of diabetes and impaired glucose tolerance in India using the WHO 1999 criteria: Prevalence of diabetes in India study (PODIS). Diabetes Res Clin Pract 2004;66:301-7. http://dx.doi.org/10.1016/j.diabres.2004.04.008 ; PMid:15609460.

46. Singh RB, Ghosh S, Niaz AM, Gupta S, Bishnoi I, Sharma JP, et al. Epidemiologic study of diet and coronary risk factors in relation to central obesity and insulin levels in rural and urban populations of North India. Int J Cardiol 1995:47:24555. http://dx.doi.org/10.1016/0167-5273(94)02186-M

47. Subramanian SV, Smith GD. Patterns, distribution, and determinants of underand overnutrition: a population-based study of women in India. Am J Clin Nutr 2006;84:633-40. PMid:16960179.

48. Chhabra P, Chhabra SK. Distribution and determinants of body mass index of non-smoking adults in Delhi, India. J Heal Popul Nutr 2007:25:294-301. PMid:18330062 PMCid:PMC2754037.

49. Sidhu S, Kumari K. Incidence of overweight and obesity among urban and rura males of Amritsar. J Exerc Sci Physiother 2006;2:79-83.

50. Shukla HC, Gupta PC, Mehta HC, Hébert JR. Descriptive epidemiology of body mass index of an urban adult population in western India. J Epidemio Community Health 2002;56:876-80. http://dx.doi.org/10.1136/jech.56.11.876 PMid:12388581 PMCid:PMC1732045

51. Kaur G, Singh SP, Singh A. Prevalence of overweight and obesity in urban and rural women of Punjab. Hum Biol Rev 2013;2:306-13.

52. Rathi MA, Irani A, Kakrani VA. Obesity and its risk factors in urban and ru- 
Singh et al:: Socio-economic Patterning of Cardiovascular Disease and its Risk Factors among Indians

ral females of Pune. IOSR J Nurs and Health Sci 2014;3:24-8. http://dx.doi. org/10.9790/1959-03532428.

53. Gopalan C. Obesity In The Indian Urban 'Middle Class'. Bull Nutr Found India 1998;19:1-5.

54. Kaur J, Kaur M. Relation of sedentary lifestyle with cardiovascular parameters in primary care patients. Cardiovas Dis 2015;3:294-300. http://dx.doi. org/10.18281/jcvd.2015.1.3.

55. Murray Cramm J, Jinkook L. Smoking, physical activity and healthy aging in India. BMC Public Health 2014;14:1349-60. http://dx.doi.org/10.1186/1471-245814-526 ; PMid:24884852 PMCid:PMC4047779

56. Sullivan R, Kinra S, Ekelund U, Bharathi AV, Vaz M, Kurpad A, et al. Socio-demographic patterning of physical activity across migrant groups in India: results from the Indian Migration Study. PLoS One 2011;6:e24898. http://dx.doi. org/10.1371/journal.pone.0024898; PMid:22022366 PMCid:PMC3194815

57. Anjana RM, Pradeepa R, Das AK, Deepa M, Bhansali A, Joshi SR, et al. Physical activity and inactivity patterns in India - results from the ICMR-INDIAB study (Phase-1) [ICMR-INDIAB-5]. Int J Behav Nutr Phys Act 2014;11:26. http://dx.doi. org/10.1186/1479-5868-11-26 ; PMid:24571915 PMCid:PMC3974063

58. Sachdeva S, Tyagi A, Sachdeva R, Nagar M, Bharti. Alcohol consumption practices amongst adult males in a rural area of Haryana. Med J Dr DY Patil Univ 2014;7:128. http://dx.doi.org/10.4103/0975-2870.126310.

59. Pednekar MS, Sansone G, Gupta PC. Association of alcohol, alcohol and tobacco with mortality: Findings from a prospective cohort study in Mumbai (Bombay) India. Alcohol 2012;46:139-46. http://dx.doi.org/10.1016/j.alcohol.2011.08.006 ; PMid:21908155.

60. Ganesh Kumar S, Premarajan KC, Subitha L, Suguna E, Kumar V. Prevalence and pattern of alcohol consumption using Alcohol Use Disorders Identification Test (AUDIT) in rural Tamil Nadu, India. J Clin Diagn Res 2013;7:1637-9.

61. Gururaj G, Girish N, Benegal V, Chandra V, Pandav R. Burden and socio-economic impact of alcohol - The Bangalore study (Alcohol Control Series No. 1). Who,South East Asia Regionl Office, New Delhi: 2006.

62. John A, Barman A, Bal D, Chandy G, Samuel J, Thokchom M, et al. Hazardous alcohol use in rural southern India: nature, prevalence and risk factors. Natl Med J India 2009;22:123-5. PMid:19764687.

63. Katyal R, Bansal R, Agrawal V, Goel K, Chaudhary V. Cross - sectional study to acknowledge the independent association of the socio - demographic determinants of alcohol use in an urban slum of north India 2014;5:749-57.

64. Pillai A, Nayak MB, Greenfield TK, Bond JC, Nadkarni A, Patel V. Patterns of alcohol use, their correlates, and impact in male drinkers: A population-based survey from Goa, India. Soc Psychiatry Psychiatr Epidemiol 2013;48:275-82. http:// dx.doi.org/10.1007/s00127-012-0538-1 ; PMid:22752108 PMCid:PMC3529206.

65. Subramanian SV, Nandy S, Irving M, Gordon D, Smith GD. Role of socioeconomic markers and state prohibition policy in predicting alcohol consumption among men and women in India: A multilevel statistical analysis. Bull World Health Organ 2005;83:829-36. PMid:16302039 PMCid:PMC2626474.

66. Neufeld KJ, Peters DH, Rani M, Bonu S, Brooner RK. Regular use of alcohol and tobacco in India and its association with age, gender, and poverty. Drug Alcohol Depend 2005;77:283-91. http://dx.doi.org/10.1016/j.drugalcdep.2004.08.022 PMid:15734228

67. Easwaran M, Bazroy J, Jayaseelan V, Singh Z. Prevalence and determinants of alcohol consumption among adult men in a coastal area of south India. Int J Med Sci Public Heal 2015;4:1. http://dx.doi.org/10.5455/ijmsph.2015.1010201479: http://dx.doi.org/10.5455/ijmsph.2015.08052015344.

68. Gupta PC, Saxena S, Pednekar MS, Maulik PK. Alcohol consumption among middle-aged and elderly men: A community study from western India. Online 2003;38:327-31. http://dx.doi.org/10.1093/alcalc/agg077.

69. Bhawna G. Burden of smoked and smokeless tobacco consumption in india - results from the global adult tobacco survey india (GATS-INDIA)- 20092010. Asian Pacific J Cancer Prev 2013;14:3323-9. http://dx.doi.org/10.7314/ APJCP.2013.14.5.3323

70. Sreelakshmi PR, Arsha NS, Vijayakumar K, Devi M, Sreelal TP, Balaji J. Mortality risk among tobacco users in a rural population of southern India : Evidence from a cohort study 2014:9-11.

71. Gupta V, Yadav K, Anand K. Patterns of tobacco use across rural, urban, and urban-slum populations in a north Indian community. Indian J Community Med 2010;35:245-51. http://dx.doi.org/10.4103/0970-0218.66877 ; PMid:20922100 PMCid:PMC2940179.

72. Garg A, Singh M, Gupta V, Garg S, Daga M, Saha R. Prevalence and correlates of tobacco smoking, awareness of hazards, and quitting behavior among persons aged 30 years or above in a resettlement colony of Delhi, India. Lung India 2012;29:336. http://dx.doi.org/10.4103/0970-2113.102812; PMid:23243347 PMCid:PMC3519019.

73. Singh A, Ladusingh L. Prevalence and determinants of tobacco use in India: Evidence from recent global adult tobacco survey Data. PLoS One 2014;9:e114073. http://dx.doi.org/10.1371/journal.pone.0114073
PMid:25474196 PMCid:PMC4256395

74. Dixit S, Ansari MA, Khan Z, Khalique N. Prevalence and predictors of tobacco use; a cross-sectional household survey in Aligarh District of Uttar Pradesh. Ind J Comm Health. 2012 Oct 6;24:203-8.

75. Chockalingam K, Vedhachalam C, Rangasamy S, Sekar G, Adinarayanan S, Swaminathan $S$, et al. Prevalence of tobacco use in urban, semi urban and rural areas in and around Chennai City, India. PLoS One 2013;8:e76005. http://dx.doi. org/10.1371/journal.pone.0076005; PMid:24098418 PMCid:PMC3788037.

76. Agrawal S, Karan A, Selvaraj S, Bhan N, Subramanian S V, Millett C. Socioeconomic patterning of tobacco use in Indian states. Int $\mathrm{J}$ Tuberc Lung Dis 2013;17:1110-7. http://dx.doi.org/10.5588/ijtld.12.0916; $\quad$ PMid:23827038 PMCid:PMC4284293.

77. Subramanian SV, Nandy S, Kelly M, Gordon D, Davey Smith G. Patterns and distribution of tobacco consumption in India: cross sectional multilevel evidence from the 1998-9 national family health survey. BMJ 2004;328:801-6. http:// dx.doi.org/10.1136/bmj.328.7443.801 PMid:15070637 PMCid:PMC383376.

78. Rani M, Bonu S, Jha P, Nguyen SN, Jamjoum L. Tobacco use in India: prevalence and predictors of smoking and chewing in a national cross sectional household survey. Tob Control 2003;12:e4. http://dx.doi.org/10.1136/tc.12.4.e4; PMid:14660785 PMCid:PMC1747786.

79. Rooban T, Elizabeth J, Umadevi K, Ranganathan K. Sociodemographic correlates of male chewable smokeless tobacco users in India: A preliminary report of analysis of national family health survey, 2005-2006. Indian J Cancer 2010;47:91. http://dx.doi.org/10.4103/0019-509X.63865PMid:20622422.

80. Prabhakar B, Narake SS, Pednekar MS. Social disparities in tobacco use in India: the roles of occupation, education and gender. Indian journal of cancer. 2012 Oct 1;49(4):401. http://dx.doi.org/10.4103/0019-509X.107747 ; PMid:23442405.

81. Sorensen G, Gupta PC, Pednekar MS. Social disparities in Tobacco use in Mumbai, India: The roles of occupation, education, and gender. Indian J Cancer 2012;49:401-9. http://dx.doi.org/10.4103/0019-509X.107747 ; PMid:23442405.

82. Bhounsule P, Bhounsule S, Essien E, Sansgiry S. Predictors of cigarette smoking behavior in a cohort of adults in India. J Behav Heal 2013:2:1. http://dx.doi. org/10.5455/jbh.20130115021517.

83. Bhan N, Srivastava S, Agrawal S, Subramanyam M, Millett C, Selvaraj S, et al. Are socioeconomic disparities in tobacco consumption increasing in India? A repeated cross-sectional multilevel analysis. BMJ Open 2012;2:e001348e001348. http://dx.doi.org/10.1136/bmjopen-2012-001348 ; PMid:23024253 PMCid:PMC3488713.

84. Nag T, Ghosh A. Cardiovascular disease risk factors in Asian Indian population: A systematic review. Journal of cardiovascular disease research. 2013 Dec 31;4(4):222-8. PMid:24653585.

85. Gupta R, Prakash H, Gupta VP, Gupta KD. Prevalence and determinants of coronary heart disease in a rural population of India. J Clin Epidemiol 1997;50:2039. http://dx.doi.org/10.1016/S0895-4356(96)00281-8.

86. Rastogi T, Vaz M, Spiegelman D, Reddy KS, Bharathi AV, Stampfer MJ, et al. Physical activity and risk of coronary heart disease in India. Int $\mathrm{J}$ Epidemiol 2004;33:759-67. http://dx.doi.org/10.1093/ije/dyh042 ; PMid:15044412.

87. Kar SS, Thakur JS, Virdi NK, JainS, Kumar R. Risk factors for cardiovascular diseases: Is the social gradient reversing in northern India? Natl Med J India 2010;23:206-9. PMid:21192513.

88. Ezzati M, Vander Hoorn S, Lawes CMM, Leach R, James WPT, Lopez AD, et al. Rethinking the "diseases of affluence" paradigm: Global patterns of nutritional risks in relation to economic development. PLoS Med 2005;2:040412. http://dx.doi.org/10.1371/journal.pmed.0020133 ; PMid:15916467 PMCid:PMC1088287.

89. Gross national income per capita, Atlas method 2011-15. [Internet] World Bank [Cited- 2016 Feb 13] Avaliable from: http://data.worldbank.org/indicator/NY.GNP. PCAP.CD

90. Sansone GC, Raute LJ, Fong GT, Pednekar MS, Quah ACK, Bansal-Travers M, et al. Knowledge of health effects and intentions to quit among smokers in India: Findings from the Tobacco Control Policy (TCP) India Pilot Survey. Int J Environ Res Public Health 2012;9:564-78. http://dx.doi.org/10.3390/ijerph9020564 ; PMid:22470310 PMCid:PMC3315264.

91. Kinra S, Johnson M, Kulkarni B, Rameshwar Sarma KV, Ben-Shlomo Y, Smith GD. Socio-economic position and cardiovascular risk in rural indian adolescents: evidence from the Andhra Pradesh children and parents study (APCAPS) Public Health 2014;128:852-9. http://dx.doi.org/10.1016/j.puhe.2014.06.005 ; PMid:25220689 PMCid:PMC4211329.

92. Sharma HK, Tripathi BM, Pelto PJ. The evolution of alcohol use in India. AIDS Behav 2010;14:8-17. http://dx.doi.org/10.1007/s10461-010-9727-7 PMid:20571859.

93. Gupta R, Gupta KD. Coronary heart disease in low socioeconomic status subjects in India: "An evolving epidemic". Indian Heart J 2009;61:358-67. PMid:20635739. 